\title{
Aging and diffusion in low dimensional environments
}

\author{
Laurent Laloux* and Pierre Le Doussal** \\ * Laboratoire de Physique Théorique et Hautes Energies, ${ }^{1} 4$ Place Jussieu, 75252 Paris Cedex 05, France \\ ** CNRS-Laboratoire de Physique Théorique de l'Ecole Normale Supérieure ${ }^{2}, 24$ rue Lhomond 75231 Paris France
}

(September 29, 2018)

\begin{abstract}
We study out of equilibrium dynamics and aging for a particle diffusing in one dimensional environments, such as the random force Sinai model, as a toy model for low dimensional systems. We study fluctuations of two times $\left(t_{w}, t\right)$ quantities from the probability distribution $Q\left(z, t, t_{w}\right)$ of the relative displacement $z=x(t)-x\left(t_{w}\right)$ in the limit of large waiting time $t_{w} \rightarrow \infty$ using numerical and analytical techniques. We find three generic large time regimes: (i) a quasi-equilibrium regime (finite $\tau=t-t_{w}$ ) where $Q(z, \tau)$ satisfies a general FDT equation (ii) an asymptotic diffusion regime for large time separation where $Q(z) d z \sim \bar{Q}\left[L(t) / L\left(t_{w}\right)\right] d z / L(t)$ (iii) an intermediate "aging" regime for intermediate time separation $\left(h(t) / h\left(t_{w}\right)\right.$ finite), with $Q\left(z, t, t^{\prime}\right)=f\left(z, h(t) / h\left(t^{\prime}\right)\right)$. In the unbiased Sinai model we find numerical evidence for regime (i) and (ii), and for (iii) with $\overline{Q\left(z, t, t^{\prime}\right)}=Q_{0}(z) f\left(h(t) / h\left(t^{\prime}\right)\right)$ and $h(t) \sim \ln t$. Since $h(t) \sim L(t)$ in Sinai's model there is a singularity in the diffusion regime to allow for regime (iii). A directed model, related to the biased Sinai model is solved and shows (ii) and (iii) with strong non self-averaging properties. Similarities and differences with mean field results are discussed. A general approach using scaling of next highest encountered barriers is proposed to predict aging properties, $h(t)$ and $f(x)$ in landscapes with fast growing barriers. It accounts qualitatively for aging in Sinai's model. We also identify a mecanism for aging in low dimensional phase space corresponding to an almost degeneracy of barriers. We illustrate this mechanism by introducing a new exactly solvable model, with barriers and wells, which shows clearly diffusion and aging regimes with a rich variety of functions $h(t)$.
\end{abstract}

\section{INTRODUCTION}

There is presently considerable interest in out of equilibrium dynamical processes. For systems without quenched disorder these are important to understand phenomena such as coarsening and domain growth ${ }^{3}$. In systems such as spin glasses ${ }^{4-8}$, random fields, interfaces, glasses in vortex systems ${ }^{12,15,13}$, which are dominated by quenched disorder and ultra slow relaxations, a detailed understanding of out of equilibrium dynamics becomes absolutely necessary to make contact with numerical simulations and experiments. These usually involve studying relaxation dynamics from an initial configuration at $t=0$ (e.g uncorrelated) and asking about correlations in the systems between two later times $t^{\prime}$ (also called $t_{w}$ the waiting time) and $t<t^{\prime}$. An interesting question to ask is what happens when both $t$ and $t^{\prime}$ are taken to infinity. Since there are of course many ways to take $t, t^{\prime}$ to infinity and one wishes to classify the possible regimes.

In out of equilibrium situations the usual properties of equilibrium dynamics do not hold. Such properties are the time translational invariance (TTI), i.e the dependence on $t-t^{\prime}$ only of the correlation functions as well as the fluctuation dissipation theorem (FDT) which relates linear response to time derivatives of correlations functions. A first question is then to ask how to take $t, t^{\prime}$ to infinity and still recover an equilibrium regime.

Glassy systems with quenched disorder were found to exhibit a variety of non equilibrium properties often generically termed "aging". Loosely it means that the properties of the system are governed by the age of the system $t_{w}$, i.e the time after the quench ${ }^{16,18,17}$ For instance it is expected that correlation functions in these systems have dependences such has $t / t_{w}$. This type of dependence is also found in simpler out of equilibrium systems such as coarsening in spin systems without disorder ${ }^{3}$ which also exhibit dependences of correlation functions of the form $L(t) / L\left(t_{w}\right)$. This dependence originates from the growth of domains of size $L(t)$ and diffusion of the domain walls. A stronger form of aging seems to be observed ${ }^{4,5}$, and was proposed for spin glasses where the linear response shows memory effects, e.g the remanent linear magnetization after applying a field during time $t_{w}$ decays very slowly over a time scale set only by $t_{w}$. Other puzzling phenomena such as memory under thermal cycling are observed ${ }^{4,6,7}$. There is at present no theory which would account fully for all these phenomena ${ }^{9}$, and understanding is only partial. Ideas and scaling arguments borrowed from domain growth and coarsening were also applied to disordered models ("droplet picture" ${ }^{8}$ ), but it is unclear whether they can account for all situations. More recently exact solutions were obtained ${ }^{19}$ for the out of equilibrium dynamics of several mean field models ${ }^{36,37}$, some of which where found to exhibit strong aging properties. It makes several non trivial predictions ${ }^{19}$ for both the correlation function $C\left(t, t^{\prime}\right)$ and the response 
function $R\left(t, t^{\prime}\right)$ of these mean-field models in the limit of large times $t, t^{\prime}$. The asymptotic time regime in these models has been successfully resolved, under some physical assumptions, and turns out to be in direct correspondence with Parisi's static replica symmetry breaking solution (with some important differences). However the matching of the small time regime to the asymptotic one remains problematic. A general picture for aging dynamics in mean field was proposed ${ }^{19}$. The resemblance with some of the features observed in spin glass experiments seems encouraging, though many points remain unclear ${ }^{20}$. There has been some attempts to classify the various aging behaviours, and to differentiate the domain growth coarsening type of "aging" from a stronger type analogous to what is found in mean field models ${ }^{10}$. Despite these recent advances, there is however at present no detailed microscopic understanding of the aging phenomena.

An elegant microscopic mechanism for aging was proposed some time ago by Feigelman and Vinokur (FV) $)^{23}$ in the context of diffusion in a one dimensional environment. Using a semi-quantitative analysis, they proposed that traps with wide distribution of waiting times (a diverging first moment $\langle\tau\rangle=\infty$ ) would naturally lead to aging phenomena and waiting time dependence. The idea is that because $\langle\tau\rangle=\infty$, at time $t_{w}$ the system is typically in a trap of release time $\sim t_{w}$ and thus the diffusing particle sees potential barriers which effectively grows with $t_{w}$. The Feigelman-Vinokur trap model was later popularized by Bouchaud ${ }^{39}$ and generalized, on the basis of previous work on wide distributions of waiting times ${ }^{28-30,33,31}$ to describe various situations, some inspired by the statics of mean field models ${ }^{41}$. It is important to note, however, that in the one dimensional Sinai model with a bias originally studied by FV and in Ref. ${ }^{29}$ the wide distributions can be shown to be dynamically generated and not artificially put by hand. Despite the phenomenological appeal of the FV mecanism for aging it is unclear how far it can be pushed ${ }^{39}$ to describe all the physics of glasses and provide a non artificial, dynamically generated mecanism for aging. In fact rather different scenarios were proposed to understand more microscopically mean field dynamics ${ }^{11}$

In this paper we study some simple one dimensional diffusion model, such as Sinai's model and investigate in some details their out of equilibrium dynamics. We will not attempt to propose any phenomenological model for aging in real glasses but simply study the extreme case of low dimensional phase space and identify the various possible large time regimes. These low dimensional models are dominated by activated dynamics over energy barriers and stand at the opposite end from mean field models. One can hope that they serve as toy models for diffusion in low dimensional space and give some insight into finite dimension. In particular, one can check whether some of the ideas introduced in mean-field theory carry through. Specifically, mean field generalizations of Sinai's model (i.e diffusion in a $d$ dimensional random potential with $d \rightarrow \infty$ ) were solved ${ }^{36}$ and can be directly compared. The advantage of this one dimensional model is that the correlation and response functions can in principle be computed numerically up to very large times. Note that a simulation of Sinai's model was performed recently in ${ }^{34}$, but with a much too slow algorithm to reach significant times, as we will discuss. A first summary of the present study was contained $\mathrm{in}^{21}$.

It becomes clear in our study that here the full probability distribution should be studied, while in mean field theory it is enough to study the second moment. For a diffusing particle the interesting quantity is the distribution $Q\left(z, t, t^{\prime}\right)$ of displacements $z=x(t)-x\left(t^{\prime}\right)$ between times $t$ and $t^{\prime}$ (the particle having started at $t=0$ at a random position) with a translational or configurational average of $Q$. The second moment $B\left(t, t^{\prime}\right)=<z^{2}>_{Q}$ was computed in mean field $^{36}$. The results are as follows for $t \rightarrow \infty$ and $t^{\prime} \rightarrow \infty$. There is:

(i) a quasi equilibrium regime for finite $\tau=t-t^{\prime}$ where TTI $\left(B\left(t, t^{\prime}\right)=B(\tau)\right)$ and FDT theorems hold. In that regime displacements are bounded since $B(\tau \rightarrow \infty)=b_{0}$.

(ii) for more separated times $B\left(t, t^{\prime}\right)>b_{0}$ keeps growing. This is the aging regime where $B\left(t, t^{\prime}\right)$ remains a fixed number $B\left(t, t^{\prime}\right)=B$ provided $t$ and $t^{\prime}$ grow in some well defined way, i.e with $h(t) / h\left(t^{\prime}\right)$ a fixed number (a function of $B$ ). In that very non trivial regime some new generalized FDT theorems hold, according to the general theory of ${ }^{19}$. The function $h(t)$ is not determined by the large time mean field solution, and at present must be determined numerically. The number $B$ can eventually be chosen as large as wanted. For large $B$ this regimes crosses over into a diffusion regime. Interestingly there is a singularity at the beginning of the aging regime with a non trivial exponent $\beta$, i.e the function $B\left(t, t^{\prime}\right)=B\left[h(t) / h\left(t^{\prime}\right)\right]$ is non analytic as a function of $h(t) / h\left(t^{\prime}\right)-1$. A singularity at the beginning of the aging regime is indeed found in a variety of experimental glassy systems. The exponent $\beta$, relevant for mode coupling theories of real glasses ${ }^{18}$, was obtained analytically in ${ }^{36}$.

Note also that in mean field there is some mathematical correspondance between two times $t, t^{\prime}$ and replica pairs $a, b$. There, roughly speaking the quasi equilibrium regime (i) was found to correspond to the replica symmetric part of the static solution, while the aging regime (ii) corresponded to the RSB part of the static solution. The correspondence is in fact not perfect because dynamical quantities do not always coincide with their static counterpart ${ }^{19,38,36}$.

It is important to know what remains of the above dynamical mean field scenario in low dimensional models, such as the models studied here, and we will attempt to give some elements of answer. Obviously this scenario will be modified since we now have to deal with full distributions $Q\left(z, t, t^{\prime}\right)$. One would like e.g. to identify possible mechanisms which can reproduce aging with non trivial functions $h(t)$ and exponents $\beta$.

We will use Sinai's model as a starting point. It is interesting because it is related to coarsening models with disorder. For instance it can model a single interface in a random field model, or motion of kinks in vortex lines, or dislocation 
loops, in presence of point disorder. The dynamics of Sinai's model has been studied extensively ${ }^{22,24,25,29,26}$. The model was shown to exhibit ultra-slow diffusion $\overline{\left\langle x(t)^{2}\right\rangle} \sim(\log t)^{4}$. The response to a driving force $f$ was also shown to be quite anomalous, with several phases. There is a threshold force such that for $f<f_{c}$ the velocity vanishes $V=0$ and diffusion is sublinear $\overline{\left\langle x(t)^{2}\right\rangle} \sim t^{2 \mu}$ with a continuously variable exponent $\mu=f / f_{c}$. For $\left.f\right\rangle f_{c}$ one has $V \sim\left(f-f_{c}\right)$ and similar transitions in higher order moments of the displacement take place at larger $f$. The physics of this anomalous response was also understood and shown to be related ${ }^{29,26}$ to the existence of barriers with an exponential distribution of energy heights, resulting in power-law distributions of trapping times and Levy distributions for first passage times.

Let us illustrate some of the questions by some simple consideration. In the case of the case of an applied bias, even the first moment of the displacement already contains interesting information. It is known ${ }^{24,29,26}$ that one has exactly, at large times $\left\langle\overline{x(t)-x(0)>}=C t^{\mu}\right.$ where $C$ depends on the details of the model. The aging nature of this expression is clear since one can write:

$$
<\overline{x(t)-x\left(t^{\prime}\right)>}=C\left(t^{\mu}-t^{\prime \mu}\right) \sim C \mu \frac{\tau}{t_{w}^{1-\mu}}
$$

for $t^{\prime}=t_{w}, t=t_{w}+\tau$. This indicates that there is an aging regime in this model since one can take $\tau \rightarrow \infty$ and $t_{w} \rightarrow \infty$ keeping $<\overline{x(t)-x\left(t^{\prime}\right)>}$ a fixed number, provided:

$$
\tau \sim t_{w}^{1-\mu}
$$

In particular, in the limit where $t_{w}$ goes to infinity first, there is no motion at all in any finite time interval! We also note that the above result can be rewritten as:

$$
\overline{<x(t)-x\left(t^{\prime}\right)>}=C \ln \frac{h(t)}{h\left(t^{\prime}\right)}
$$

where $h(t)=\exp \left(t^{\mu}\right)$. This form (3) (with $h(t)$ unspecified) is of the type found in large time solutions of mean field models as mentionned above. Similarly, subaging forms for $h(t)=\exp \left((\ln t)^{a}\right)$ with $a>1$, as observed in experiments $^{20}$, could in principle originate from logarithmic diffusion process. Indeed one can write:

$$
\ln ^{a} t-\ln ^{a} t^{\prime}=\ln \frac{h(t)}{h\left(t^{\prime}\right)} \sim \frac{\tau}{\left(t_{w} / \ln ^{a-1} t_{w}\right)}
$$

with the observed $h(t)$. One would thus like to investigate these analogies further.

We will thus first study Sinai's model in Section II (symmetric model) and in Section III (directed model). Then we will give in Section IV a general discussion of barrier mechanisms for aging which we believe allow to understand the results obtained on Sinai's model. We introduce a method to estimate aging functions by looking at the sequences of next highest barriers. Finally, in Section V we introduce and solve a model based on these considerations which exhibits non trivial againg and diffusion behaviour.

\section{SYMMETRIC SINAI MODEL}

\section{A. the model}

In this Section we study the one dimensional Sinai model ${ }^{26,22,24,25,27,29}$. In its continuous version it is described by the Langevin equation:

$$
\frac{d x}{d t}=F(x(t))+\eta(t)
$$

where $<\eta(t) \eta\left(t^{\prime}\right)>=2 T \delta\left(t-t^{\prime}\right)$ is the thermal noise and $\overline{F(x) F\left(x^{\prime}\right)}=\sigma \delta\left(x-x^{\prime}\right)$ is a quenched random force which is gaussian and uncorrelated. It may have an average $\overline{F(x)}=f$. Writing $F(x)=-d U(x) / d x$ it describes the thermal motion of a particle in a one dimensional gaussian random potential landscape $U(x)$. The landscape $(x, U(x))$ can be seen itself as a trajectory of a random walker ( $x$ playing then the role of the time). In the general case $f>0$ the lanscape is tilted (the walker experiences a bias) but we will only consider here the case $f=0$ (symmetric Sinai model). Then the random potential has long range correlations:

$$
\overline{(U(x)-U(y))^{2}}=\sigma|x-y|
$$


Thus barriers grow with scale which results in an anomalously slow dynamics $<(x(t)-x(0))^{2}>\sim(\ln t)^{4}$. The $N$ dimensional version of this model (i.e $x=\left(x_{1}, x_{2}, x_{N}\right)$ ) was solved in the limit $N \rightarrow \infty$ in Ref. ${ }^{36}$.

In this Section we study a space discretized version of Sinai's model which can be easily studied numerically. It is expected that this discretized version has the same large time physics as the continuous model (this was shown for some quantities in the weak disorder limit ${ }^{28,29}$. It is defined by a Fokker-Planck (FP) equation:

$$
\frac{d P_{n}(t)}{d t}=\left(H_{F P}\right)_{n m} P_{m}(t)=W_{n, n+1} P_{n+1}(t)+W_{n, n-1} P_{n-1}(t)-\left(W_{n+1, n}+W_{n-1, n}\right) P_{n}(t)
$$

where $P_{n}(t)$ is the probability that a particle is at site $n$ at time $t$ (with some initial condition at $t=0$ ). The hopping rates $W_{n+1, n}, W_{n-1, n}$ are quenched random variables. It is convenient to parametrize these rates as in Ref. ${ }^{29}$ in the form:

$$
W_{n-1, n}=e^{-\phi_{n}} \quad W_{n, n-1}=e^{\phi_{n}}
$$

This describes effectively the Arrhenius diffusion of a particle on a one dimensional lattice in a random potential $U_{n}=-2 \sum_{k=0}^{n} \phi_{k}$. There is in effect a force $2 \phi_{n}$ on the link between site $n-1$ and $n$. The equilibrium solution of equation 7 corresponding to zero link current $J_{n, n-1}=W_{n, n-1} P_{n-1}-W_{n-1, n} P_{n}=0$ is $P_{n}^{e q}=e^{-U_{n}}$. Here temperature is set to $T=1$. In the discrete Sinai model the variables $\phi_{n}$ are chosen gaussian independent from site to site, with $\overline{\phi_{n} \phi_{n^{\prime}}}=\sigma / 4 \delta_{n n^{\prime}}$ and the random potential thus follows a gaussian discrete random walk as a function of $n$, with correlations $\overline{\left(U_{n}-U_{n^{\prime}}\right)^{2}}=\sigma\left|n-n^{\prime}\right|$.

The important quantity to determine is the Green function $P\left(n, t \mid n_{0}, t_{0}\right)\left(t \geq t_{0}\right)$ of the FP operator $H_{F P}$ in a given environment. It is defined as the solution of (7) with initial condition $P\left(n, t_{0} \mid n_{0}, t_{0}\right)=\delta_{n n_{0}}$.

We have computed numerically $P\left(n, t \mid n_{0}, t_{0}\right)$ using exact diagonalization of the corresponding Schrödinger operator on a finite size ring. This operator is a tridiagonal symmetric matrix which can be easily diagonalized for large size $L$. The method, as well as the expressions for the correlation and response functions, is detailed in the Appendix A. We use $L+1$ sites $k=0, L$ with both reflexive and periodic boundary conditions. We have used up to $L=250$ sites and averages over $10^{3}$ and $510^{3}$ disorder configurations. Because Sinai's diffusion is so slow, we were able to study times up to $10^{15}$ without spurious effects (edges, precision). We used $\sigma=2$ in all simulations and checked the consistency of the results with several random number generators.

From this exact expression one can average either one or a product of two of these Green functions to obtain single time or two times quantities, respectively. For finite times, translational averages (with e.g uniform initial measure in an infinite single environment) and averages over disorder should coincide. Note that this will not be correct in a very special case studied below of a periodic environment. We now describe the results and their interpretation using simple arguments.

\section{B. single time quantities}

Averaged single time quantities can be obtained from the disorder averaged Green function $\overline{P\left(n, t \mid n_{0}, 0\right)}$. There are some exactly known results for this single time quantities and we will thus start by comparing with these results (as a check of our simulations).

$\mathrm{In}^{28}$ the averaged probability density at the origin $\overline{P\left(n_{0}, t \mid n_{0}, 0\right)}$ was computed exactly in the continuum limit for all times $t$. It describes the weak disorder universal behaviour $\left(\mathrm{see}^{29}\right)$. At large $t$ the complete scaling form for the averaged

diffusion front, i.e the distribution of the variable $n /(\log t)^{2}$ was obtained in $^{44}$ as $\overline{P(n, t \mid 0,0)} \simeq(\log t)^{-2} p\left[n(\log t)^{-2}\right]$. The function $p(x)$ is (up to a constant rescaling):

$$
p(x)=\frac{2}{\pi} \sum_{k=0}^{+\infty} \frac{(-1)^{k}}{2 k+1} \exp \left(-\frac{(2 k+1)^{2} \pi^{2}}{8}|x|\right)
$$




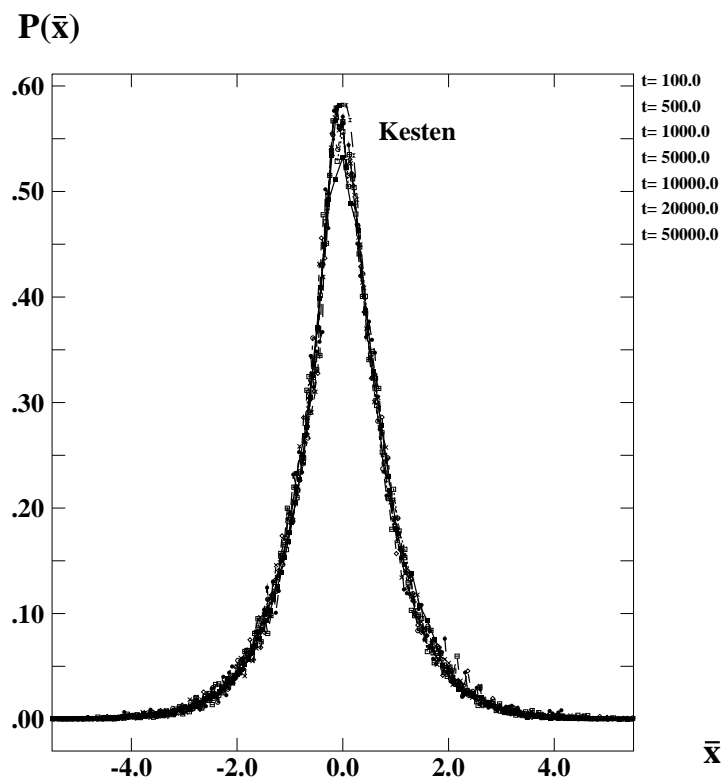

FIG. 1. Single time averaged diffusion front: at large time our simulations cannot be distinguished from Kesten's prediction. We denote $\bar{x}=x / x_{r m s}(t)$. $L=125$ and $510^{3}$ configurations.

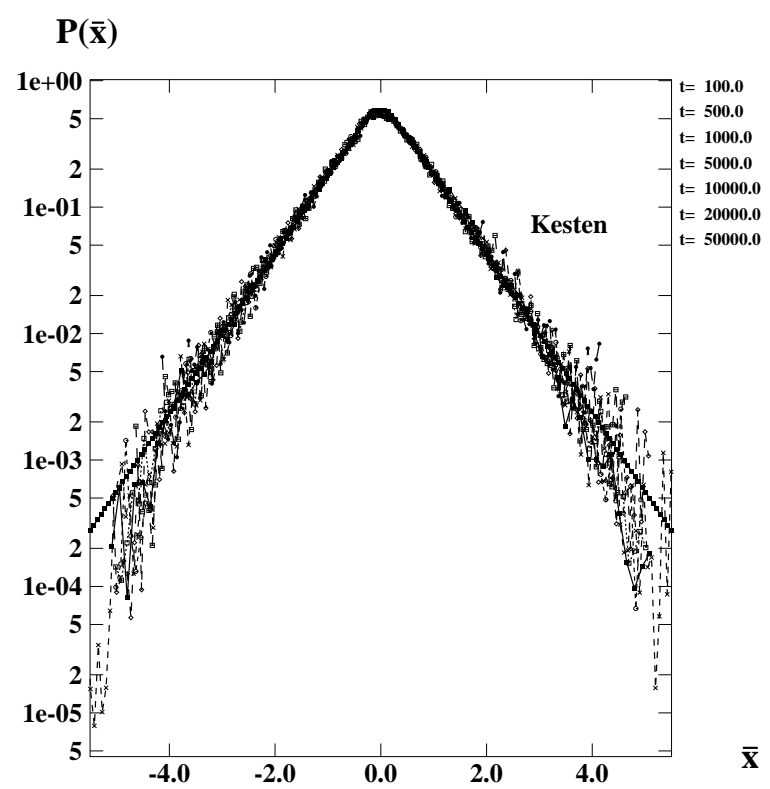

FIG. 2. Same as Fig. 1. in logarithmic scale for $P$

We have computed numerically $\overline{P(n, t \mid 0,0)}$ and the $x \sim(\log t)^{2}$ law as a check of our program. In Fig. 1 and Fig. 2 we have plotted the scaling function $p[x]$ as it is determined numerically and the analytic result of Kesten (we have fixed the scale by imposing equality of the second moment and we denote $x_{r m s}=\sqrt{\overline{\left\langle x^{2}(t)>\right.}}$ ). One can see that the agreement is excellent. It improves considerably on an earlier determination by Nauenberg ${ }^{45}$. The behaviour $x \sim\left(\log \left(t / t_{0}\right)\right)^{2}$ is plotted in Fig. 3 and also agrees. We have not attempted to fit the amplitude to known results (we have checked that the order of magnitude is correct) since we were satisfied with the agreement in Fig. 1. 


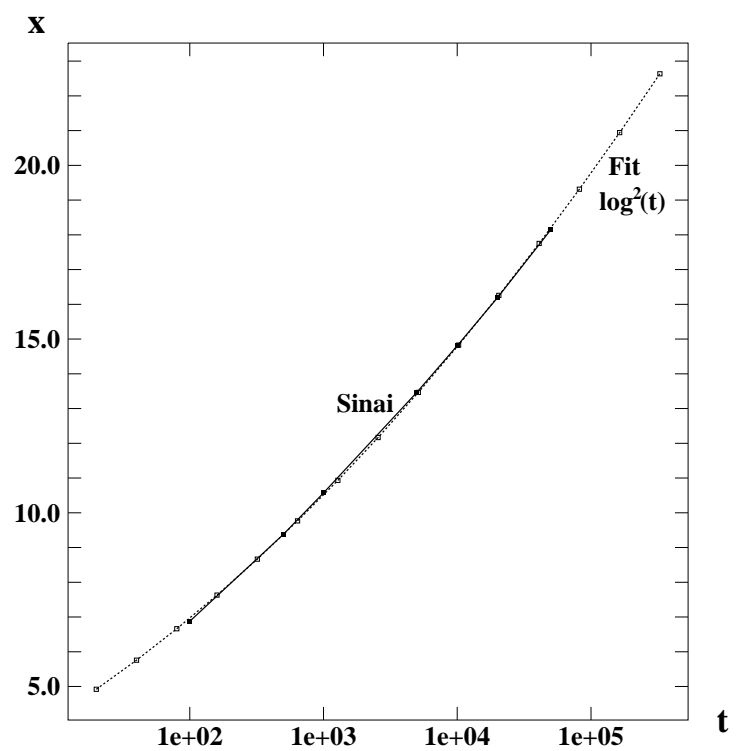

FIG. 3. Numerical data compared to Sinai's diffusion law $x \sim x_{r m s}(t) \sim \log \left(t / t_{0}\right)^{2} . L=125$ and $510^{3}$ configurations.

We now turn to two time quantities.

\section{C. two time quantities}

There are no analytical results available at present, to our knowledge for two time quantities. This make the numerical simulation all the more important.

We have computed numerically the two time averaged Green function: $\overline{P\left(n, t \mid n^{\prime}, t^{\prime}\right) P\left(n^{\prime}, t^{\prime} \mid n_{0}, t_{0}=0\right)}$. It is a complicated object which is difficult to analyze. Thus we have started by computing, as in mean field models, the configurationally averaged mean squared displacement.

$$
B\left(t, t^{\prime}\right)=\overline{\left\langle\left(x(t)-x\left(t^{\prime}\right)\right)^{2}\right\rangle}=\sum_{n, n^{\prime}}\left(n-n^{\prime}\right)^{2} \overline{P\left(n, t \mid n^{\prime}, t^{\prime}\right) P\left(n^{\prime}, t^{\prime} \mid n_{0}, t_{0}=0\right)}
$$

The best way is to plot it as a function of $t-t^{\prime}$ for different waiting times $t_{w}=t^{\prime}$. It is represented in Fig. 4 One sees that it clearly does not depend only on $t-t^{\prime}$ and that, the larger the waiting time, the slower it grows. The dynamics slows down considerably as $t^{\prime} \rightarrow \infty$. This is very reminiscent of what happens in mean field models. One also sees that an effective plateau develops as $t^{\prime}$ becomes larger. In mean field it would be a true plateau ${ }^{36}$ which defines the Edwards Anderson order parameter $b_{E A}$. Thus here an important question is whether there is a finite limit for $B\left(t_{w}+\tau, t_{w}\right)$ as $t_{w} \gg \tau \gg 1$ as in the mean field model. 


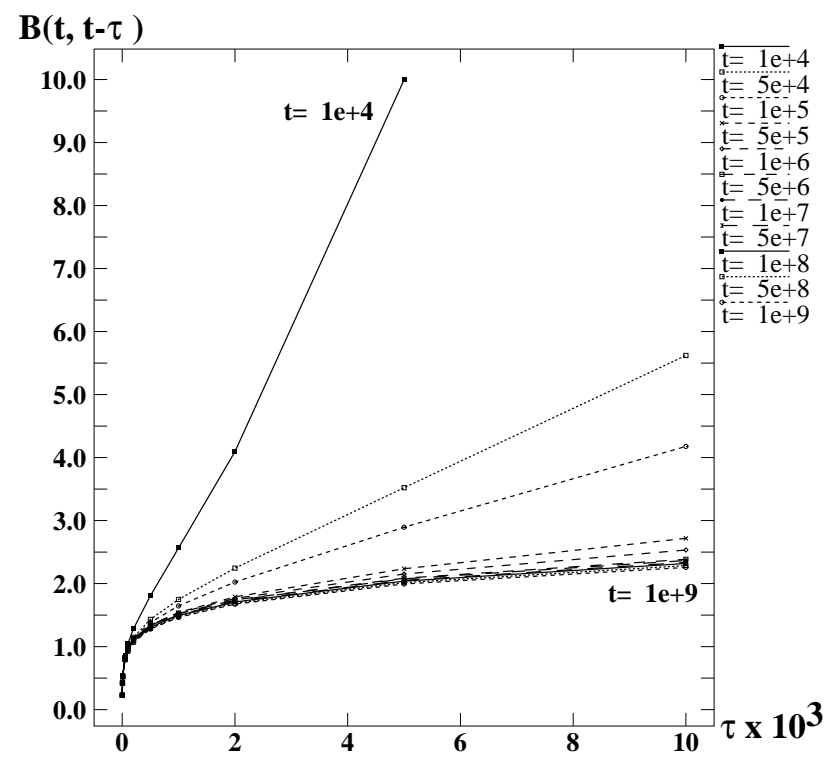

FIG. 4. mean squared separation $B\left(t=t_{w}+\tau, t_{w}\right)$ as a function of $\tau$ for several $t \sim t_{w}$. A limit curve $B(\tau)$ can be seen, but $B(\tau)$ keeps growing with $\tau$ while in mean field it would go to a constant $b_{E A}=B(\tau=\infty)$. $L=125$ and $10^{3}$ configurations.

The answer to this question is negative. This is because, as will be discussed below, in finite dimension contrary to mean field, while the distribution of $x(t)-x\left(t^{\prime}\right)$ converges towards a limit, it second cumulant is infinite. In fact studying only the second cumulant $B\left(t_{w}+\tau, t_{w}\right)$ is of little help in the present problem. One needs to study the full disorder averaged distribution of relative displacements:

$$
\overline{Q\left(z, t, t_{w}\right)}=\int d x^{\prime} \overline{P\left(x^{\prime}+z, t \mid x^{\prime}, t_{w}\right) P\left(x^{\prime}, t_{w} \mid 0, t_{0}=0\right)}
$$

and not only its second cumulant. We have found that when looking at that distribution, several regimes can be identified.

\section{D. quasi-equilibrium regime}

The first question to ask is whether there is a quasi equilibrium regime when $t$ and $t_{w}$ are close together. It can be defined, for instance as $t=t_{w}+\tau$ with $\tau$ fixed and finite (and $t_{w} \rightarrow \infty$ ). In mean field this would correspond to the FDT regime, where time translational invariance and the fluctuation dissipation theorem are found to hold.

One can thus ask whether the following limit distribution exists:

$$
\overline{Q(z, \tau)}=\lim _{t_{w} \rightarrow \infty} \lim _{L \rightarrow \infty} \overline{Q\left(z, t_{w}+\tau, t_{w}\right)}
$$

where $L$ is the size of the system and whether in that limit, some equilibrium theorems hold.

We are not able to answer to this important question rigorously in all generality and we encourage other workers to do so. We will however provide some elements which we hope will shed some light on the issues.

One strategy is to first study the case of an infinite periodic medium but with a very large period $L_{0}$ (i.e almost disordered). There some things can be shown when $t_{w}$ is very large. Of course this is cheating a bit since the idea would be to take $L_{0} \rightarrow \infty$ eventually, and thus this is like interverting the limit $t_{w} \rightarrow \infty$ and $L_{0} \rightarrow \infty$. It does give some insight though. Thus we will then check, using numerics and physical arguments, whether it can be extended to a non periodic case.

\section{1. a periodic model}

We are considering an infinite periodic medium. We assume that the potential $U(x)$ is periodic which corresponds to the situation with no drift. One can generalize to the case with a bias by taking a periodic force (using the results of $^{46}$. We will first consider a single environment (no configurational averages). 
We are interested in the distribution:

$$
Q_{L_{0}}\left(z, t_{w}+\tau, t_{w}\right)=\int_{-\infty}^{+\infty} d x^{\prime} P_{L_{0}}\left(x^{\prime}+z, t_{w}+\tau\left|x^{\prime} t_{w}\right| x_{0} 0\right)
$$

It can be written as a sum over periods:

$$
\begin{aligned}
& Q_{L_{0}}\left(z, t_{w}+\tau, t_{w}\right)=\sum_{k} \int_{0}^{L_{0}} d x^{\prime} P_{L_{0}}\left(x^{\prime}+k+z, t_{w}+\tau \mid x^{\prime}+k, t_{w}\right) P_{L_{0}}\left(x^{\prime}+k, t_{w} \mid x_{0} 0\right) \\
& =\int_{0}^{L_{0}} d x^{\prime} P_{L_{0}}\left(x^{\prime}+z, \tau \mid x^{\prime}, 0\right) \tilde{P}_{L_{0}}\left(x^{\prime}, t_{w} \mid x_{0} 0\right)
\end{aligned}
$$

where we have defined the periodized Green function:

$$
\tilde{P}_{L_{0}}\left(x^{\prime}, t^{\prime} \mid x_{0} 0\right)=\sum_{k} P_{L_{0}}\left(x^{\prime}+k L, t^{\prime} \mid x_{0} 0\right)
$$

and we have used the periodicity of the disorder $P_{L_{0}}\left(x, t \mid x^{\prime}, t^{\prime}\right)=P_{L_{0}}\left(x+m L, t \mid x^{\prime}+m L, t^{\prime}\right)$. So, up to now it is exact for any configuration of the disorder.

Since the periodized function $\tilde{P}$ is in fact the Green function of the problem on a ring of size $L_{0}\left(\right.$ see e.g $\mathrm{g}^{22,46}$ ), it converges towards the equilibrium Gibbs measure on the ring in the large $t_{w}$ limit:

$$
\lim _{t_{w} \rightarrow \infty} \tilde{P}_{L_{0}}\left(x^{\prime}, t_{w} \mid x_{0} 0\right)=\frac{e^{-U\left(x^{\prime}\right) / T}}{\int_{0<x^{\prime}<L_{0}} d x^{\prime} e^{-U\left(x^{\prime}\right) / T}}
$$

This implies that:

$$
Q_{L_{0}}(z, \tau) \rightarrow \int_{0}^{L_{0}} d x^{\prime} P_{L_{0}}\left(x^{\prime}+z, \tau \mid x^{\prime} 0\right) \frac{e^{-U\left(x^{\prime}\right) / T}}{\int d y e^{-U(y) / T}}
$$

This is particularly useful when $\tau$ is fixed and much smaller than the time necessary to travel a period (i.e $(\ln \tau)^{2} \ll$ $L_{0}$ in Sinai's model). This formula can also be generalized in presence of a drift using the stationary distribution obtained $\mathrm{in}^{46}$. Note that these arguments extend to any finite dimensional problem.

\section{2. non periodic case}

In the non periodic case, when $L_{0} \rightarrow \infty$ before $t_{w}$, it is obvious that for a single configuration of disorder there is no limit to $Q\left(z, t_{w}+\tau, t_{w}\right)$ as $t_{w} \rightarrow \infty$. Indeed numerically Sinai's diffusion consists of sudden jumps to deeper and deeper wells. Thus even though in each successive well there is presumably a quasi-equilibrium regime $Q\left(z, t_{w}+\tau, t_{w}\right) \sim$ $Q_{\text {well }}(z, \tau)$ it will depend on the details of each new well encountered. In some sense there is a distribution of such quasi equilibrium distributions (which is not unlike the image from replica symmetry breaking). In addition, there are times $t_{w}$ where the packet jumps, but they presumably become more and more rare at large $t_{w}$. Thus only the disorder averaged (or translational average) can be expected to converge to some limit $\overline{Q(z, \tau)}$, as these features will get smoothed out. We will assume that this convergence holds (based on our numerical evidence).

One possible further assumption is that once averaged over disorder one has:

$$
\overline{Q(z, \tau)}=\overline{Q_{e q}(z, \tau)} \equiv \lim _{L_{0} \rightarrow \infty} \overline{Q_{L_{0}}(z, \tau)}
$$

where $Q_{L_{0}}(z, \tau)$ is the distribution in the periodized medium (using the same disorder distribution) given in (18). We have called $Q_{e q}(z, \tau)$ this distribution.

A possible reasonable starting assumption is that they are equal in $\bar{Q}=\overline{Q_{e q}}$ in Sinai's model. We do have good numerical indication that this is indeed correct, as shown in Fig. 5. However, because differences, if they exist, could be subtle, small and hard to detect we emphasize that whether this is strictly correct should be checked further (there are indeed cases where it is wrong - see the solvable model of Section V). In any case, the above equation provides at least a basis for comparison. 


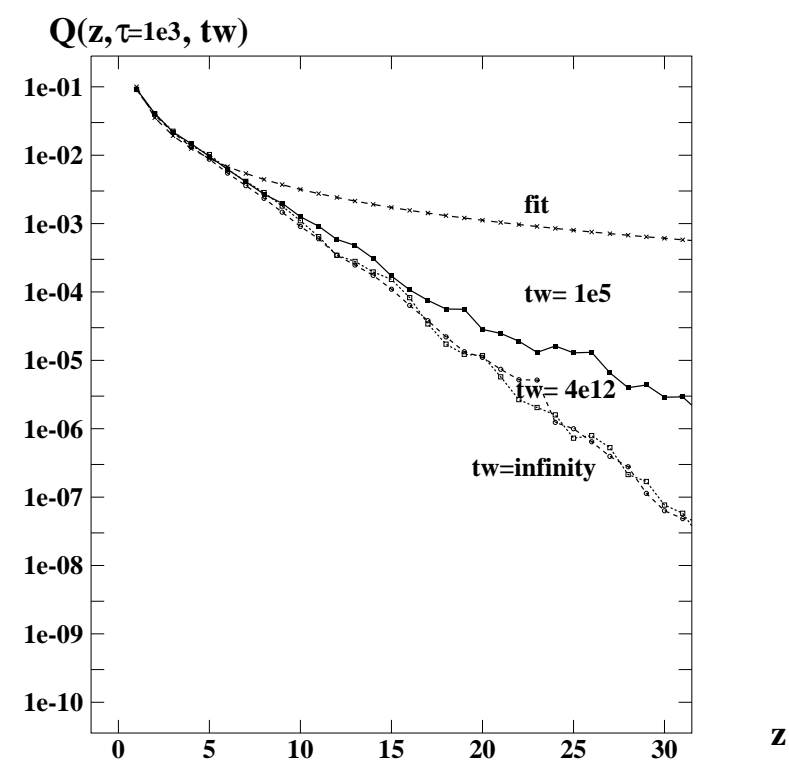

FIG. 5. Convergence, for a fixed $\tau$, of $\overline{Q\left(z, t_{w}+\tau, t_{w}\right)}$ (represented here for $t_{w}=10^{5}$ and $\left.t_{w}=410^{12}\right)$ towards $\overline{Q_{e q}(z, \tau)}$ computed here from formula $(18,19)$ (curve called " $t_{w}=$ infinity" in the figure). The limit curve $\overline{Q(z, \tau=\infty)}$ is shown by comparison (curve called "fit"). $L=200$ and $510^{3}$ configurations.

\section{3. correspondence with the statics}

Let us investigate the large $\tau$ limit of $\overline{Q(z, \tau)}$. One expect, if the above is assumption is correct, that:

$$
\overline{Q(z, \tau)} \sim \lim _{L_{0} \rightarrow \infty} \overline{Q_{L_{0}}(z, \tau)} \rightarrow \lim _{L_{0} \rightarrow \infty} \overline{Q_{L_{0}}(z)}=\lim _{L_{0} \rightarrow \infty} \overline{\int_{0}^{L_{0}} d x^{\prime} \frac{e^{-U\left(x^{\prime}+z\right) / T} e^{-U\left(x^{\prime}\right) / T}}{\left(\int d y e^{-U(y) / T}\right)^{2}}}
$$

Thus the two time calculation becomes a two replica correlation function in a replica calculation.

\section{properties of the quasi static distribution}

For Sinai model one can argue that there is indeed a limit averaged quasi static distribution $\bar{Q}(z)$. From the above formula (20) and since there is typically one global minimum which dominates the period $L_{0}$ in Sinai's landscape, it is clear that this distribution will consist of two parts:

(i) a part localized near $z=0$ of finite extent, with a finite weight.

(ii) a part which is strongly non self averaging with, for each environment, some localized peaks around some $z_{i}$. Averaging over disorder yields a smooth $Q(z)$ with algebraic tail at large $z$ :

$$
Q(z) \sim \frac{A}{z^{3 / 2}}
$$

This tail comes from configurations of the disorder which look as in Fig. 6. The lowest well $U_{\min }$ in the sample of period $L_{0}$ is represented and it happens that there is a secondary well, with a bottom at $U_{\min }+E_{n}$ with $E_{n}=O(T)$, at a finite distance $z_{0}$ of the first one. Then roughly the measure $Q(z)$ will consist of two peaks localized around the two wells.

Since in Sinai model the random potential can itself be seen as performing a random walk, the probability that such an environment occurs can be estimated from the probability of return to the origin of a random walk. This yields a probability $\sim z_{0}^{-3 / 2}$ and thus the above algebraic tail of $Q(z)$. More refined calculation, taking into account that the principal well is an absolute minimum can be performed and lead to estimates for $A$, but go beyond this paper. Let us point out that the above equilibrium Gibbs measure has been analyzed recently in Ref. ${ }^{47}$ and they also obtained analytically the above algebraic tail (i.e formula (21)). This tail however can be explained from simple arguments.

It would be interesting to check whether one can extend ideas from mean field and whether in a replica calculation of (20) the localized part near the origin would correspond to the replica symmetric part of the solution while the tails from the rare events to the part with broken replica symmetry. 
So there is also an Edwards Anderson order parameter here but it is a distribution. Because of non self averaging effects one needs the full distribution $Q(z)$. It would be nice to check more rigorously that $\overline{Q(z)}=\overline{Q_{e q}(z)}($ and thus that there is no extra dynamical order parameter).

Finally let us emphasize that the above arguments for the tail of the quasi equilibrium distribution are very similar to static droplet model arguments ${ }^{8}$. Here however they are made in a dynamical context.

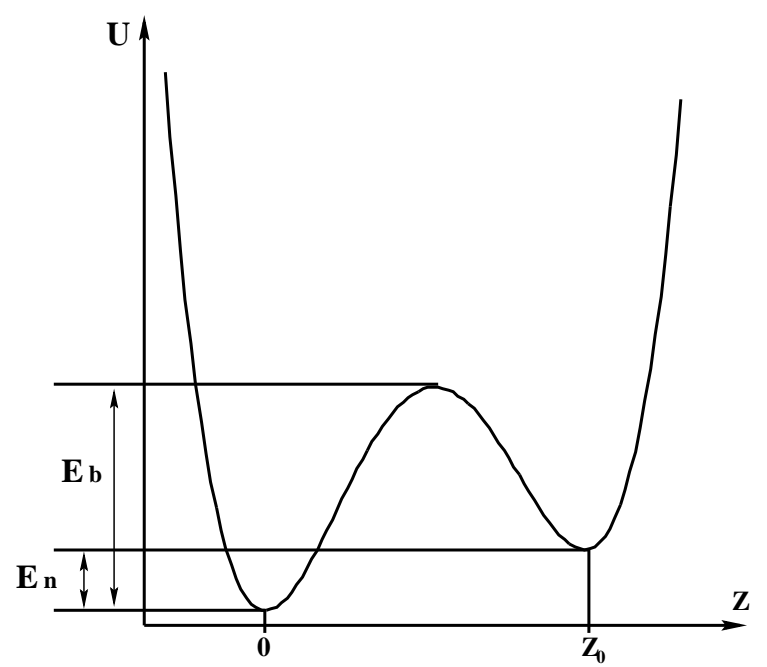

FIG. 6. Two well (FDT-TTI) quasi equilibrium regime: rare configurations leading to sample fluctuations and algebraic decay of $Q(z)$

The above arguments thus suggest that the moments of the relative displacements:

$$
\lim _{t_{w} \rightarrow \infty} \overline{<\left(x\left(t_{w}+\tau\right)-x\left(t_{w}\right)\right)^{n}>} \sim \int z^{n} Q(z, \tau)
$$

converge towards a finite limit when $\tau \rightarrow \infty$ only for $n<1 / 2$. For $n>1 / 2$ they grow unboundedly with $\tau$. These moments growing with $\tau$ may not a priori be incompatible with being in a quasi equilibrium state (though it calls for further investigations). In mean field, this does not happen since even for unbounded diffusion problems ${ }^{36}$ the quasi equilibrium regime is such that $B\left(t, t^{\prime}\right)<b_{E A}$.

We now estimate these moments (22) using a simple two well model taking into account the crossing of the barrier $E_{b}$ between the two wells. Let us write:

$$
Q(z, \tau)=\delta(z) \frac{1}{2}\left(1+e^{-\tau e^{-E_{b}}}\right)+\delta\left(z-z_{0}\right) \frac{1}{2}\left(1-e^{-\tau e^{-E_{b}}}\right)
$$

and average over $z_{0}$ and the barrier height which we can take to scale as $E_{b}\left(z_{0}\right) \sim \alpha z_{0}^{1 / 2} \alpha$ being a positive random variable. This yields the moments:

$$
\lim _{t_{w} \rightarrow \infty} \overline{<\left(x\left(t_{w}+\tau\right)-x\left(t_{w}\right)\right)^{n}>} \sim \frac{1}{2} \int d z z^{n} \frac{A}{z^{3 / 2}}\left(1-e^{-\tau e^{-\alpha z^{1 / 2}}}\right)
$$

Introducing the scaled variable $y=z / \ln ^{2} \tau$ and using that the function $\left(1-e^{-e^{-\alpha \ln ^{2} \tau\left(y^{1 / 2}-1 / \alpha^{2}\right)}}\right) \approx \theta\left(1 / \alpha^{2}-y\right)$ one finds, for $n>1 / 2$.

$$
\lim _{t_{w} \rightarrow \infty} \overline{<\left(x\left(t_{w}+\tau\right)-x\left(t_{w}\right)\right)^{n}>} \sim A(\ln \tau)^{2 n-1}
$$




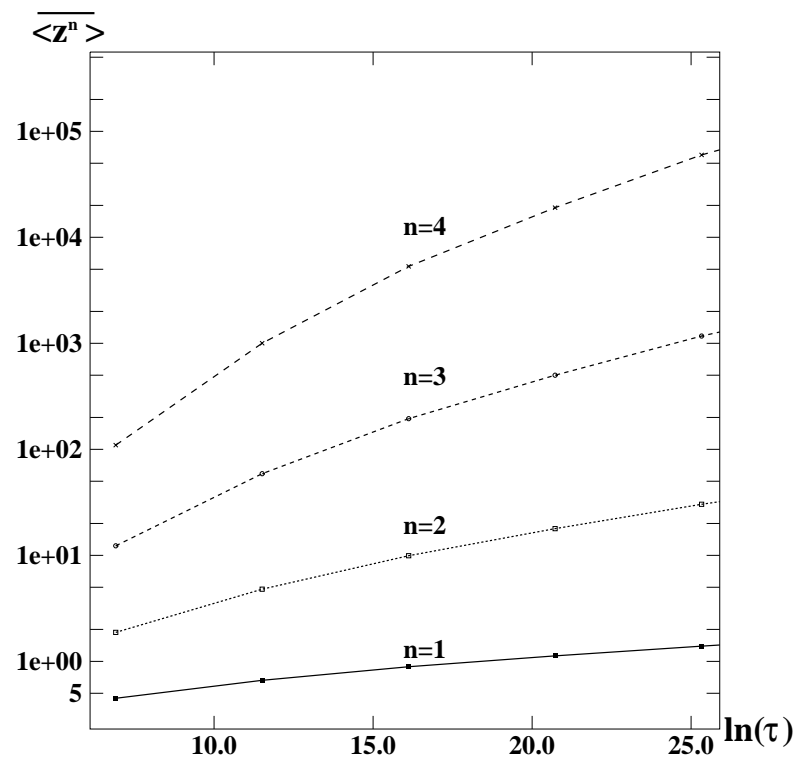

FIG. 7. Numerical determination of the moments $\left\langle|z|^{n}(\tau)\right\rangle_{Q}$ as a function of $\ln \tau$. $L=200$ and $510^{3}$ configurations.

We have plotted these moments as determined numerically in Fig. 7. The logarithmic growth of moments $n>1 / 2$ is clearly demonstrated (we have checked that e.g. $n=1 / 4$ saturates at large $\tau$ ). Though they seem to follow (25) qualitatively, a more quantitative agreement is probably difficult to reach numerically - since these effects entirely come from rare events !

The above considerations also predict that the distribution $\overline{Q(z, \tau)}$ should converge towards $\overline{Q(z)}$ with a $z / \ln ^{2} \tau$ scaling behaviour. This is consistent with our simulations as can be seen in Fig. 8 where we have shown the $\overline{Q_{e q}(z, \tau)}$ for various $\tau$. It does indeed converge towards a limit curve $\overline{Q_{e q}(z)}$ which was well fitted by its asymptotic behaviour $\sim z^{-3 / 2}$.

One can also check from this simple model (23) that as a consequence, the Edward Anderson "overlap" parameter $\overline{Q(z=0, \tau)}$ should also converge towards it equilibrium value as $\tau \rightarrow \infty$ with $1 /(\ln \tau)^{a}$ corrections. This indeed happens as is shown in Fig. 9 .

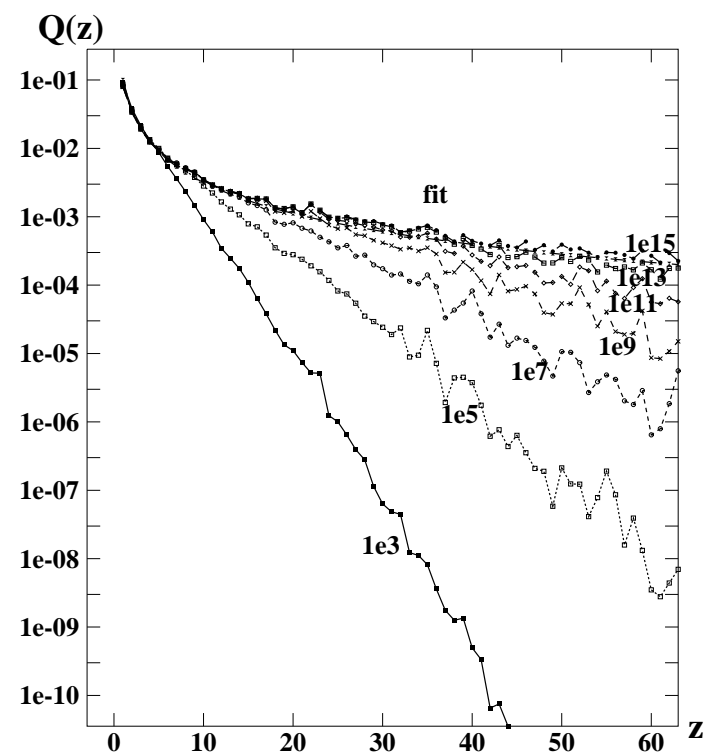

FIG. 8. Numerical determination of $\overline{Q_{e q}(z, \tau)}$ compared to the predicted asymptotic behaviour $\sim 1 / z^{(3 / 2)}$ (fit).L=200 and $510^{3}$ configurations. 


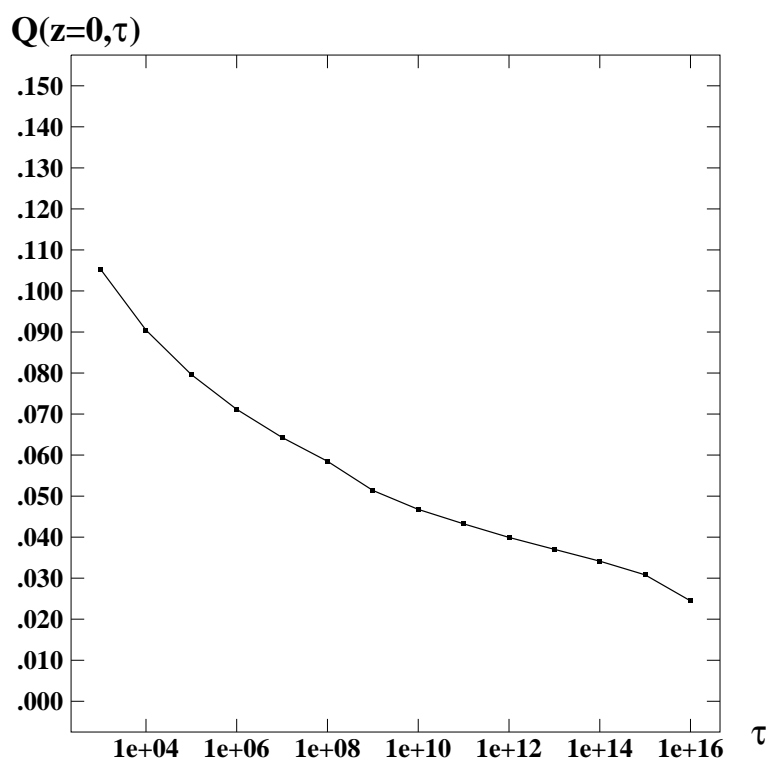

FIG. 9. Plot of $\overline{Q_{e q}(z=0, \tau)}-\overline{Q_{e q}(z=0, \tau=\infty)}$ against $\tau$ (in $\ln \tau$ scale). $L=200$ and $510^{3}$ configurations.

\section{4. equilibrium theorems}

It is plausible that for large $t_{w}$ equilibrium theorems like FDT hold for disorder averaged quantities. These provide relations between correlation functions and linear response functions.

It is useful to generalize these theorems to the full probability distribution $\bar{Q}\left(z, t, t^{\prime}\right)$. This is done in the Appendix $\mathrm{A}$ and $\mathrm{B}$ to which we refer for details. One defines:

$$
\overline{R\left(z, t, t^{\prime}\right)}=\frac{\delta \overline{Q_{h}\left(z, t, t^{\prime}\right)}}{\delta h\left(t^{\prime}\right)}
$$

where $\overline{Q_{h}\left(z, t, t^{\prime}\right)}$ is the probability when an additional infinitesimal uniform field pulse $h(t)=h \delta\left(t-t^{\prime}\right)$ is applied at time $t^{\prime}$. A technical detail is that it can be applied at time $t^{\prime}-\epsilon$ (which defines $\left.R^{+}\left(z, t, t^{\prime}\right)\right)$ or at time $t^{\prime}+\epsilon$ (which defines $R\left(z, t, t^{\prime}\right)$ and corresponds to the Ito prescription for the response, i.e $\delta<x\left(t^{\prime}\right)>/ \delta h\left(t^{\prime}\right)=0$ ). If quasi equilibrium hold (meaning the current $J$ at $t_{w}$ vanishes, see Appendix B, the time translational invariant averaged functions $\bar{Q}(z, \tau)$ for large $t^{\prime}=t_{w}$ and fixed $\tau$ and $\bar{R}(z, \tau)$ should verify the exact differential relation:

$$
-\partial_{\tau} \overline{Q(z, \tau)}=T \partial_{z} \overline{R(z, \tau)}
$$

These relations can be generalized to discrete models as done in A. The response $R^{+}(z, \tau)$ satisfies a slightly different equation:

$$
-\partial_{\tau} \overline{Q(z, \tau)}=-T \partial_{z}^{2} \overline{Q(z, \tau)}+T \partial_{z} \overline{R^{+}(z, \tau)}
$$

We determined both the distribution $\overline{R^{+}(z, \tau)}$ and $\overline{R(z, \tau)}$ numerically (as explained in $\mathrm{B}$ ). The response $\overline{R^{+}(z, \tau)}$ is plotted in Fig. 10. Note that for $\tau=\infty$ the above implies simply:

$$
\overline{R^{+}(z, \tau)}=\partial_{z} \overline{Q(z, \tau)}
$$

and we checked that $\overline{R^{+}(z, \tau)}$ has the form $\sim 1 / z^{(5 / 2)}$ expected from the above relation and $(21)$. 


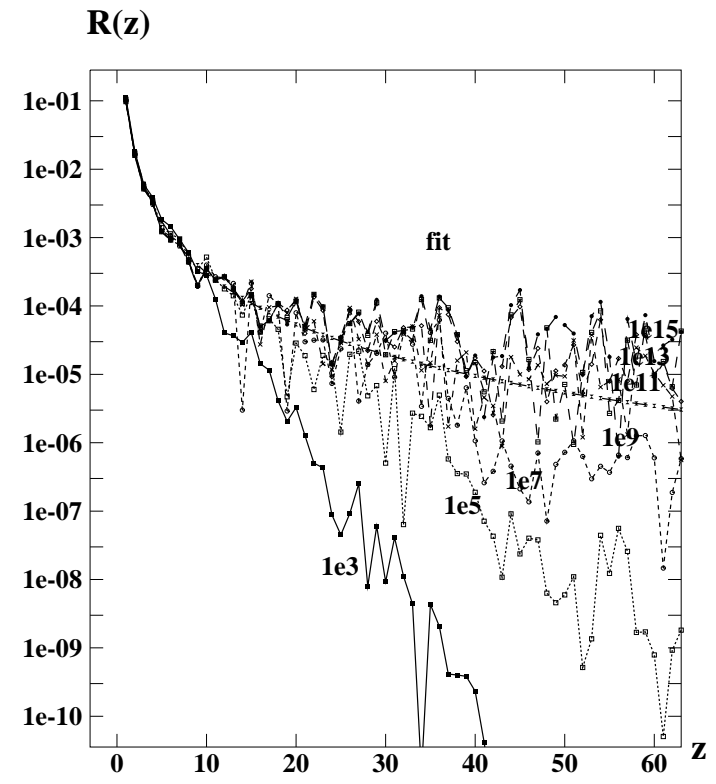

FIG. 10. Plot of $\overline{R^{+}(z, \tau)}$ ( $y$ coordinate) versus $z$ for various $\tau$ and comparison with the expected large $\tau$ limit $1 / z^{(5 / 2)}$ ("fit"). $L=200$ and $510^{3}$ configurations.

The Ito response $\overline{R(z, \tau)}$ satisfies $(27)$ and is thus analogous to a probability current. Examining $\overline{R(z, \tau)}$ obtained from our simulation as a function of $z$ confirms the above arguments based on the two well model: there is a (statistical) current flowing from the center region $z \sim 0$ to the $z>0$ (and to the $z<0$ ). In each disorder configuration $R(z=0, \tau$ ) does not have to vanish (since a each local environment is not symmetric) but its average $\overline{R(z=0, \tau)}$ must vanish by symmetry. We observe that in the simulation it is of the order $10^{-3}$ smaller than $\overline{R(z=1, \tau)}$ which is consistent.

We have plotted $\overline{R(z=5, \tau)}$ in Fig. 11. It shows clearly a $1 / \tau$ decay. To be more accurate, we have plotted $\tau \ln \tau \overline{R(z=5, \tau)}$ in Fig. 12. It indicates that:

$$
\overline{R(z, \tau)} \sim \frac{1}{\tau(\ln \tau)^{b}}
$$

with $b \approx 1$ (for a fixed $z$ ). This could be consistent with a decay of $Q(z, \tau) \sim 1 / \ln ^{2} \tau$ for fixed $z$ and a scaling $z \sim \ln ^{2} \tau$ in (27). However, $Q$ and $R$ are expected to consist of two parts with $\tau$ dependent relative weights, one part scaling as $z / \ln ^{2} \tau$ and a fixed part in $z$. Thus more work is needed to determine these functions more precisely.

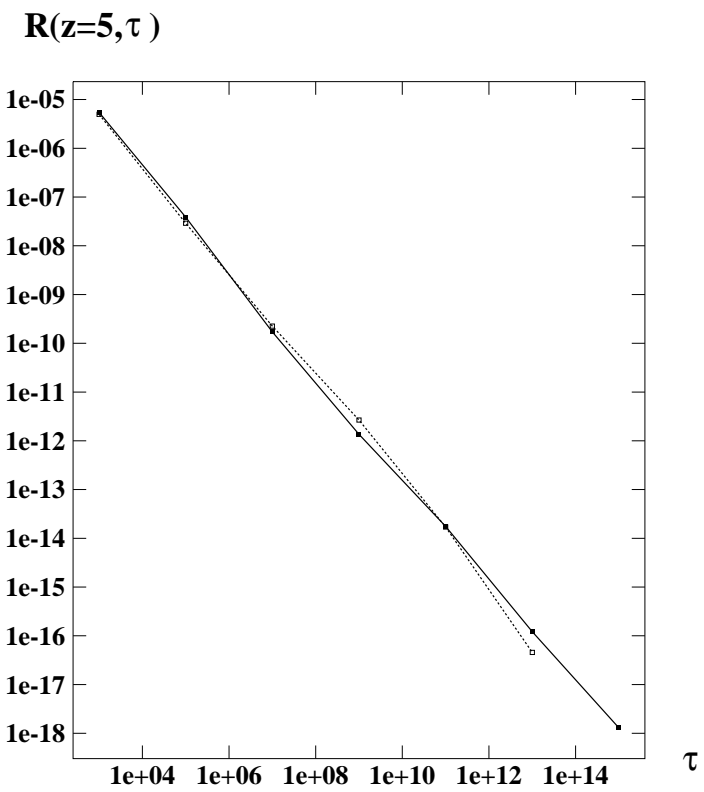


FIG. 11. Plot of $\overline{R(z=5, \tau)}$ ( $y$ coordinate) as a function of $\tau$. Two simulations are indicated: $L=100$ and $510^{2}$ configurations and $L=200$ and $10^{3}$ configurations

\section{$\mathbf{R}(\mathrm{z}=5, \tau) \log (\tau) \tau$}

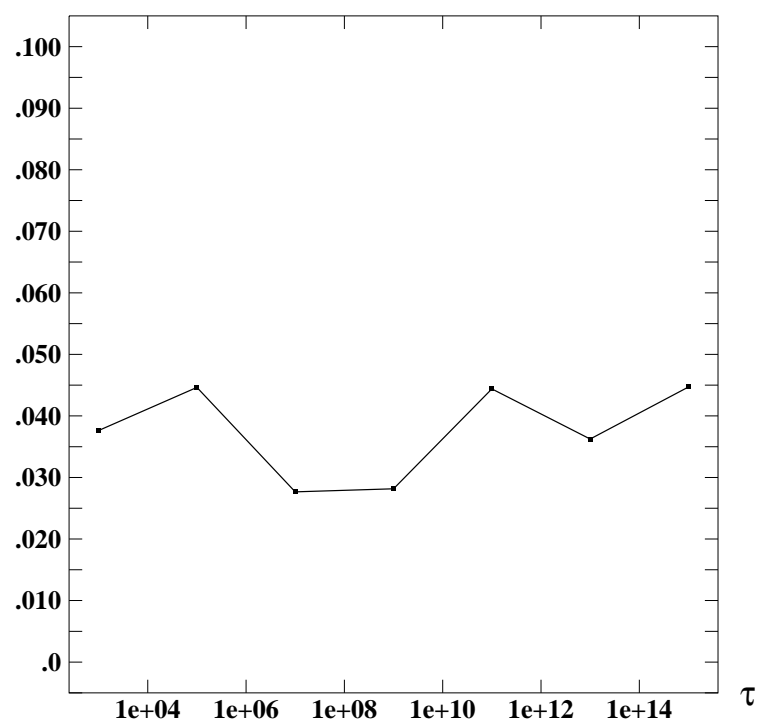

FIG. 12. Plot of $\tau \log (\tau) \overline{R(z=5, \tau)}$ ( $y$ coordinate) as a function of $\tau$. $L=200$ and $10^{3}$ configurations.

Very recently (while this manuscript was in completion) a promising approach was developped by Cugliandolo, Dean and Kurchan $(\mathrm{CDK})^{40}$ to obtain bounds which may permit to demonstrate that these quasi-equilibrium regimes exist in models such as Sinai's. Their approach is explained in Appendix C and some extensions and applications are given. For Sinai's model the bounds should be performed on disorder averaged quantities (since single environment ones do not converge). Doing this one can obtain two bounds of interest for Sinai's model:

$$
\left.\left|\overline{<x^{2}(t)>}-\overline{<x(t) x\left(t^{\prime}\right)>}-T \int_{t^{\prime}}^{t} R\left(t, t^{\prime}\right)\right| \leq \overline{\left(<x^{2}(t)>\right.}\right)^{1 / 2} \int_{t^{\prime}}^{t} d s\left(\left|\frac{d \bar{H}(s)}{d s}\right|^{1 / 2}\right.
$$

and:

$$
\left|\partial_{t^{\prime}} B\left(t, t^{\prime}\right)+2 T R\left(t, t^{\prime}\right)\right|<B\left(t, t^{\prime}\right)^{1 / 2}\left|\frac{d \bar{H}\left(t^{\prime}\right)}{d t^{\prime}}\right|^{1 / 2}
$$

The first one was given in ${ }^{40}$ (though the problem of disorder averaging is not discussed) while the second is new. $B\left(t, t^{\prime}\right)$ is defined in (10) and $R\left(t, t^{\prime}\right)=\delta \overline{<x(t)>} / \delta h\left(t^{\prime}\right)=\int d z z \overline{R\left(z, t, t^{\prime}\right)}$ (with Ito's definition of the response).

The function $\bar{H}\left(t^{\prime}\right)=\overline{\int d x^{\prime} P\left(x^{\prime} t^{\prime} \mid 00\right)\left(T \ln P\left(x^{\prime}, t^{\prime} \mid 00\right)-U\left(x^{\prime}\right)\right)}$ is the averaged free energy which satisfy an $H$ theorem (it is always decreasing). The nice observation of ${ }^{40}$ is that since in Sinai's model $\bar{H}\left(t^{\prime}\right) \sim-\ln t^{\prime}$ the above bound (31) implies that the r.h.s bound for the integrated FDT violation is $(\ln t)^{2}\left(t^{1 / 2}-t^{1 / 2}\right)$. Clearly for $\tau=t-t^{\prime}$ fixed it implies that (integrated) FDT holds, but it does even seem to imply that it holds further even for $\tau \sim t^{\prime 1 / 2}$.

Note that the second bound (32) immediately implies that if there is a limit distribution $\lim _{t_{w} \rightarrow \infty} \overline{Q\left(z, t_{w}+\tau, t_{w}\right)}$ then it will depend only on $\tau=t-t^{\prime}$ (TTI) and the bound imply that FDT is verified (since $d H\left(t^{\prime}\right) / d t^{\prime}$ goes to zero). (Note that this is also the case for the Brownian motion).

While these results are as inescapable as rigorous bounds, an explanation for this could be looked for in our previous arguments about periodic media. Indeed if our assumption (19) is correct, then one can choose $\ln ^{2} \tau \ll L_{0} \ll \ln ^{2} t_{w}$ and still have equilibrium and FDT (since the probability of having another accessible absolute minimum in the box $L_{0}$ at distances $\gg 1$ is vanishingly small (see )). Thus it is likely that in fact FDT will hold beyond what is shown by the bound, probably until $\tau \sim t_{w}^{c}$ with $c<1$ (beyond that one enters the diffusion and aging regime, see next section).

Note also for completeness the two single time bounds:

$$
\left|\partial_{t^{\prime}} \overline{<x\left(t^{\prime}\right)>}\right| \leq\left|\frac{d \bar{H}\left(t^{\prime}\right)}{d t^{\prime}}\right|^{1 / 2}
$$




$$
\left|\partial_{t^{\prime}} \overline{<x\left(t^{\prime}\right)^{2}>}\right| \leq\left|\overline{\left\langle x\left(t^{\prime}\right)^{2}>\right.}\right|^{1 / 2}\left|\frac{d \bar{H}\left(t^{\prime}\right)}{d t^{\prime}}\right|^{1 / 2}
$$

Thus we have found that an (equilibrium) TTI diffusion regime for the process $z(\tau)$ persists within the quasi equilibrium regime.

One can also apply these bounds to the case with a an applied force $f \sim \mu$, anticipating a little on the next Section III where the directed model will be discussed. Let us assume that $\bar{H}\left(t^{\prime}\right) \sim-<x(t)>\sim-\left(t^{\prime}\right)^{\mu}$. Then (31) leads to:

$$
\overline{\left\langle<x^{2}(t)>\right.}-\overline{<x(t) x\left(t^{\prime}\right)>}-T \int_{t^{\prime}}^{t} R\left(t, t^{\prime}\right) \mid \leq t^{\mu}\left(t^{(\mu+1) / 2}-\left(t^{\prime}\right)^{(\mu+1) / 2}\right)
$$

Expanding for $\tau \ll t^{\prime}$, one finds that the l.h.s must be smaller than $\tau / t^{\prime(1-3 \mu) / 2}$. Thus it shows the existence of an FDT regime for $\mu>0$ in Sinai's model. The r.h.s goes strictly to zero as long as $\mu<1 / 3$ but if one actually divide the r.h.s by the expected scale of the l.h.s $\left(\sim t^{2 \mu}\right)$ one gets $\tau / t^{\prime(1+\mu) / 2}$. Thus a FDT regime (with $X=1$ ) seems to be possible for $\mu<1$.

This may seem surprising at first, since for $\mu>0$ the system is driven. However it makes sense physically. Remember that for $\mu<1$ the particles will spend most (all for $t_{w} \rightarrow \infty$ ) of their time in a well of release time $\sim t_{w}$ (see section III). There they have time to equilibrate. Thus in some sense the fact that there is aging (and broad distributions of release time) is intimately related to the fact that there can be equilibrium within a well.

The above bounds also put constraints on the possible aging forms for a large class of models. This will be discussed in Section (IV).

\section{E. Aging and diffusion regime}

We will now present our results for $\overline{P\left(n, t \mid n^{\prime}, t^{\prime}\right) P\left(n^{\prime}, t^{\prime} \mid n_{0}, t_{0}=0\right)}$ in the regime where $t$ and $t^{\prime}$ are large and well separated. As stressed above the data is complicated to analyze. We have found numerical evidence for two regimes.

(i) the diffusion regime

The first one is the diffusion regime where $z \sim(\ln t)^{2}$ and $\ln t^{\prime} \sim \ln t$ :

$$
\overline{Q\left(z, t, t^{\prime}\right)} \sim \frac{1}{(\ln t)^{2}} F\left[\frac{z}{(\ln t)^{2}}, \frac{\ln t^{\prime}}{\ln t}\right]
$$

In that regime the three relative displacements $x(t)-x\left(t^{\prime}\right), x(t)-x(0)$ and $x\left(t^{\prime}\right)-x(0)$ are of the same order of magnitude.

(i) the aging regime

Another regime was found by looking at the decay of $Q\left(z \sim 0, t, t^{\prime}\right)$ for $z$ fixed and small (near $z=0$ ) and $t$ and $t^{\prime}$ large. By $z$ fixed and small we mean in our numerical simulation $n=n^{\prime}$ but it means more generally that $n$ is in a finite neighborhood of $n^{\prime}$. For instance we have found that the decay of $Q\left(z=0, t, t^{\prime}\right)$ could be as slow as desired by taking both $t$ and $t^{\prime}$ to infinity. We have tried various dependences of the form:

$$
\overline{Q\left(z=0, t, t_{w}\right)} \sim f\left[\frac{h\left(t_{w}\right)}{h(t)}\right]
$$

The best fit was obtained for:

$$
h(t) \sim \ln t
$$

This is illustrated in Fig. 13 where we have used $t_{w} \sim t^{a}$ with $0 \leq a<1$ and varied $t$ with fixed $a$ which is consistent with $h(t)=\ln t$, since $a=\ln t_{w} / \ln t$. For $a=1$ one recovers $\overline{Q_{e q}(z=0)}$ as expected. Note that it is more difficult numerically to get correctly the small $a$ behaviour (hence the curvature of the curves for small $a$ on Fig. 13 which is due to the smallness of the available $t_{w} \sim t^{a}$ ). We have also tried $t_{w} \sim t / 2$ in order to test a possible $t / t_{w}$ dependence of this quantity (upper curve). This curve is in fact indistinguishable at large $t$ from $\overline{Q_{e q}(z=0)}$. This strongly indicate that aging with a form $t / t_{w}$ is unlikely.

We want to emphasize that we could not rule out other forms more complicated than $h(t)=\ln t$. However, the form $t / t_{w}$ appears very unlikely for the measured quantity (e.g we haven't checked the behaviours of the moments). 


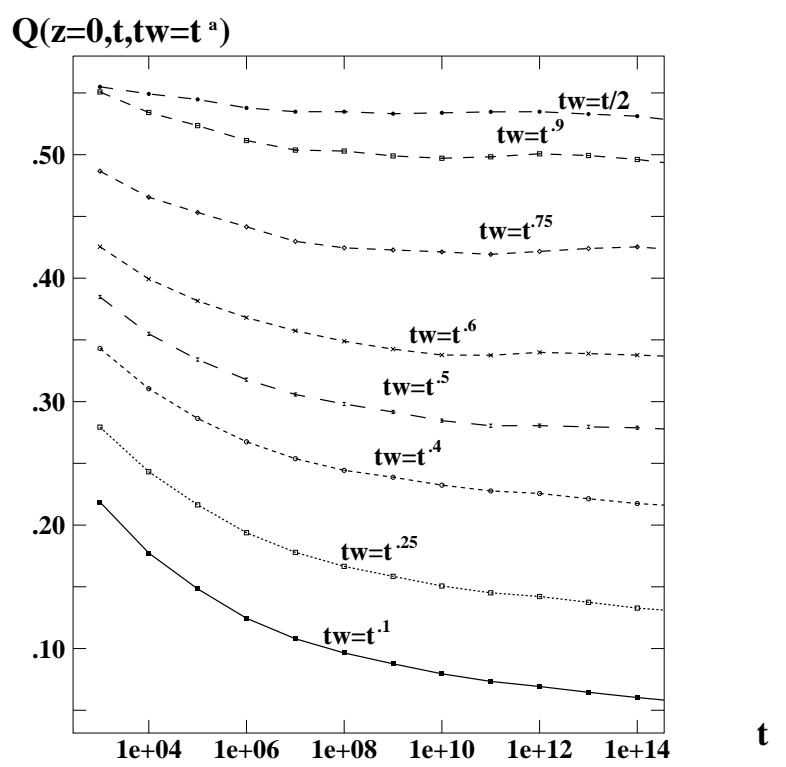

FIG. 13. Plot of $\overline{Q\left(z=0, t, t^{a}\right)}$ for various values of $a$ which indicates that the aging scaling function is $h(t) \sim \ln t$. The curvature of the curves for small $a$ at times not very large is due to the smallness of $t^{a}$. $L=200$ and $510^{3}$ configurations.

We have also computed $\overline{Q\left(z, t, t^{a}\right)}$ for several $z$ fixed and large $t$. We find that the aging behaviour is also valid for $z \neq 0$. In fact we find strong evidence for the behaviour:

$$
\overline{Q\left(z, t, t^{\prime}\right)}=Q_{0}(z) f\left[\frac{\ln t^{\prime}}{\ln t}\right]
$$

as is shown in Fig. 14 for $0 \leq z \leq 10$, where we have been able to collapse all the various curves $\overline{Q\left(z, t, t^{\prime}\right)}$ on the same curve $Q_{0}(z)$. Thus this curve should also be equal to $Q_{0}(z)=Q_{e q}(z, \tau=\infty) / Q_{e q}(z=0, \tau=\infty)$.

This behaviour seems to be consistent with a picture of aging in Sinai's model (at least for small $z$ ) where an equilibrated packet jumps as a whole out of its large well when $t^{\prime} \sim t^{a}$. This will be discussed further in Section IV. Though the collapse in Fig. 14 is perfect at small $z$ (better than $10^{-4}$ in relative precision) it becomes poorer at larger $z$. This is probably due to the fact that for the available times one overlaps somehow with the diffusion regime. It is unlikely, but not ruled out, that this could be the sign of yet another regime (more work would be necessary).

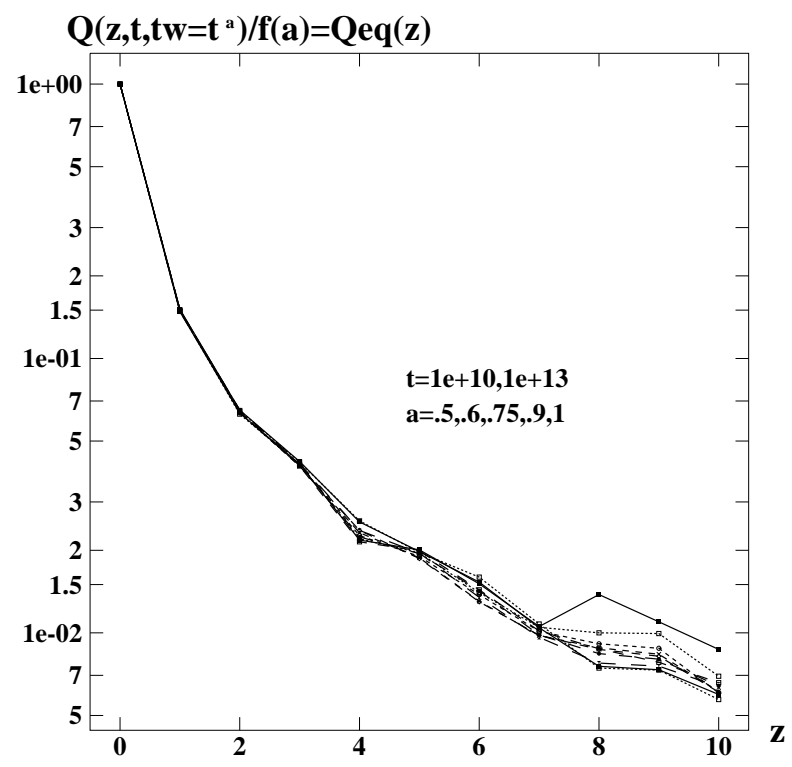


FIG. 14. Plot of $\overline{Q\left(z, t, t^{a}\right)} / \overline{Q\left(z=0, t, t^{a}\right)}$ as a function of $z$ for several values of $a$ and for two times $t=10^{10}$ and $t=10^{13}$. $L=250$ and $10^{3}$ configurations.

The fact that the temporal scaling is found to be the same $h(t) \sim L(t) \sim \ln t$ in both this aging regime and the diffusion regime indicate that these two regimes may in fact be the same in this model. If that is indeed correct it does imply that the above two time diffusion scaling function (36) must have a singularity near the origin, e.g a delta function singularity. This remains to be investigated further.

Note that in mean field, in short range models, one can also see that the diffusion scale and the aging scales actually coincide $^{36}$. Here it seems that the same happens, though for a different quantity $Q\left(z \sim 0, t, t^{\prime}\right)$.

\section{DIRECTED MODEL}

In this Section we study aging and diffusion in a simple model of directed diffusion with disorder where two time correlation functions as well as response functions can be computed simply. This model will appear as a particular case of a more general 'solvable model' which we will introduce in Section V

In this model the particle can only jump to the right of the occupied site and quenched disorder is introduced by choosing at each site $n$ an average waiting time $\tau_{n}$ according to a given distribution $P(\tau)$. The distribution of waiting times is further chosen with an algebraic tail at large times $P(\tau) \sim C / \tau^{1+\mu}$. This directed model was introduced and studied (for single time quantities) in ${ }^{33}$. It was also shown ${ }^{33,29}$ that it is a large scale effective description of Sinai model in presence of a bias. The idea is that in presence of an applied force $f$ the potential landscape $(x, U(x))$ is a biased random walk. There are thus some places where the walker goes back against the bias. This leads to rare barriers against the drift of size $E_{b}$ with probability $\exp \left(-f E_{b}\right)$. Since the waiting time in these traps behaves as $\exp \left(-E_{b} / T\right)$ this is enough to generate dynamically an algebraic distribution of waiting times ${ }^{29}$. Whether this model is also a good description of the biased Sinai model for two times quantities remain to be investigated in details.

The model is defined by the Fokker-Planck (FP) equation:

$$
\frac{d P_{n}(t)}{d t}=H_{n m} P_{m}(t) \equiv W_{n-1} P_{n-1}(t)-W_{n} P_{n}(t)
$$

The rates at each site correspond to a mean waiting time $\tau_{n}=1 / W_{n}$.

The Green function $P\left(n, n_{0} \mid t-t_{0}\right)$ is defined as the solution of $(40)$ with initial condition $P\left(n, n_{0} \mid 0\right)=\delta_{n n_{0}}$. Its Laplace Transform (LT) $P\left(n, n_{0} \mid s\right)=\int_{0}^{+\infty} P\left(n, n_{0} \mid t\right) e^{-s t}$ can be computed easily from (40). It reads:

$$
P\left(n, n_{0} \mid s\right)=\frac{1}{s+W_{n}} \prod_{k=n_{0}}^{n-1} \frac{W_{k}}{s+W_{k}}
$$

Averaged single time averaged quantities are easily computed from:

$$
\begin{aligned}
& \overline{P(0,0 \mid s)}=\overline{\frac{1}{s+W}} \equiv \Phi(s) \\
& \overline{P(n, 0 \mid s)}=\Phi(s)(1-s \Phi(s))^{n-1}
\end{aligned}
$$

The long time limit is then obtained from the small $s$ behaviour of the function $\Phi(s)$ :

$$
\begin{gathered}
\Phi(s) \sim C \pi /(\sin \mu \pi) s^{\mu-1} \quad \mu<1 \\
\Phi(s) \sim 1 / V+C \pi /(\sin \mu \pi) s^{\mu-1} \quad 1<\mu<2 \\
\Phi(s) \sim 1 / V-D s+C \pi /(\sin \mu \pi) s^{\mu-1} \quad \mu>2
\end{gathered}
$$

This yields the several phases of the model: the subdiffusive phase $0<\mu<1$ where $x \sim t^{\mu}$ (and zero velocity), the anomalous dispersion phase $1<\mu<2$ where there is a velocity $V>0$ but dispersion is anomalous $D=\infty$, and the diffusive phase $\mu>2$. We will be mostly interested here in the case $0<\mu<1$. There the above result immediately yields a Levy diffusion front ${ }^{33}$.

We are now interested in the averaged probability that the particle advances by $m$ between $t^{\prime}$ and $t=t^{\prime}+\tau$ : 


$$
Q\left(m, \tau, t^{\prime}\right)=\left\langle\overline{\delta\left(x(t)-x\left(t^{\prime}\right)-m\right)}\right\rangle=\sum_{n \geq 0} \overline{P(n+m, n, \tau) P\left(n, 0, t^{\prime}\right)}
$$

This quantity is computed in the Appendix F. The result in Laplace variables is the following:

$$
\begin{aligned}
Q\left(m=0, s_{1}, s_{2}\right) & =\frac{\Phi\left(s_{1}\right)-\Phi\left(s_{2}\right)}{\left(s_{2}-s_{1}\right) s_{2} \Phi\left(s_{2}\right)} \\
p\left(m \geq 1, s_{1}, s_{2}\right) & =\frac{s_{2} \Phi\left(s_{2}\right)-s_{1} \Phi\left(s_{1}\right)}{\left(s_{2}-s_{1}\right) s_{2} \Phi\left(s_{2}\right)} \Phi\left(s_{1}\right)\left(1-s_{1} \Phi\left(s_{1}\right)\right)^{m-1}
\end{aligned}
$$

where $s_{1}$ is associated to $\tau$ and $s_{2}$ to $t^{\prime}$. In fact one notices that

$$
Q\left(m \geq 1, s_{1}, s_{2}\right)=\left(\frac{1}{s_{1} s_{2}}-p\left(m=0, s_{1}, s_{2}\right)\right) \Phi\left(s_{1}\right)\left(1-s_{1} \Phi\left(s_{1}\right)\right)^{m-1}
$$

and thus the disorder averages factorize:

$$
Q\left(m \geq 1, \tau, t^{\prime}\right)=\left(1-Q\left(0, \tau, t^{\prime}\right)\right) * \overline{P(m-1,0 \mid \tau)}
$$

where the $*$ denotes convolution over the variable $\tau$. This could be expected from the markovian and directed nature of the walk. The clock is set back to 0 when they exit the trap. Thus for two times quantities all one needs to know is the two time averaged probability of not moving between $t_{w}$ and $t_{w}+\tau$ plus the averaged probability of diffusing by $m$ during $\tau$.

Let us first examine the probability of no motion. Explicit laplace inversion is simple on the asymptotic form for $\mu<1$ and it gives:

$$
Q\left(m=0, \tau t^{\prime}\right)=\frac{\sin (\pi \mu)}{\pi} \int_{0}^{t^{\prime} / t} d x(1-x)^{-\mu} x^{\mu-1}=F\left(\tau / t^{\prime}\right) \quad \mu<1
$$

where:

$$
F(z)=\frac{\sin (\pi \mu)}{\pi} \int_{z}^{\infty} d y \frac{1}{y^{\mu}(1+y)}
$$

One has:

$$
F(z) \sim \frac{\sin (\pi \mu)}{\mu \pi} z^{-\mu} \quad z \rightarrow \infty
$$

and $1-F(z) \sim z^{1-\mu}$ at small $z$.

It has a aging form as a function of $\tau / t_{w}$. This is the manifestation of the Feigelman Vinokur trap model mechanism of aging. This expression is similar to the one obtained $i^{41}$ in an infinite range model. In the present case however we are also interested in the diffusion regime which we now analyze.

We see on the above expression that if $\tau$ is finite and $t_{w} \rightarrow \infty$ the probability of being trapped is 1 . There is no motion on finite time scales. We note that this directed model is not rich enough to contain information about the dynamics inside traps. The quasiequilibrium regime is thus degenerate $Q(z, \tau)=\delta_{z, 0} \delta(\tau)$.

The above result also shows that at time $t_{w}+\tau$ the fraction of particules released by the well is of order $\left(\tau / t_{w}\right)^{1-\mu}$ and that the particles which are released do fast motion (as if the clock is then set back to 0 when they exit the trap). In this model the later motion is not slower. Thus they will move typically by $\delta x \sim \tau^{\mu}$.

Thus can easily estimate the moments:

$$
\overline{<\left(x\left(t_{w}+\tau\right)-x\left(t_{w}\right)\right)^{n}>} \sim\left(\tau / t_{w}\right)^{1-\mu} \tau^{n \mu}
$$

Note that this gives, for the first moment:

$$
\overline{<\left(x\left(t_{w}+\tau\right)-x\left(t_{w}\right)\right)>} \sim \tau / t_{w}^{1-\mu} \sim\left(t_{w}+\tau\right)^{\mu}-t_{w}^{\mu}
$$

in the regime $\tau<<t_{w}$ consistent with the known result for the first moment.

We note that there is, in some sense an "aging regime" for each moment. Indeed one can impose that $\overline{<\left(x(t)-x\left(t^{\prime}\right)\right)^{n}>}$ is a fixed number while taking both $t_{w} \rightarrow \infty$ and $\tau \rightarrow \infty$ provided: 


$$
\tau \sim t_{w}^{\frac{1-\mu}{1+(n-1) \mu}}
$$

This depends on the moment itself. This illustrates the strong non self averaging properties in one dimension.

In addition to a self averaging aging regime which is confined to $z=0$ there is in this model a diffusion regime for $z \sim t^{\mu}$ and $t \sim t^{\prime}$. It can be written as:

$$
\overline{Q\left(z, t, t^{\prime}\right)}=\frac{1}{t^{\mu}} F\left[\frac{z}{t^{\mu}}, \frac{t^{\prime}}{t}\right]
$$

Using the above exact relation:

$$
\overline{Q\left(z, t, t^{\prime}\right)}=\overline{Q\left(z=0, t, t^{\prime}\right) P\left(z, t-t^{\prime}, 0\right)}
$$

where $P(x, t, 0)$ is the single time diffusion front which takes a scaling form $P(x, t, 0) \sim t^{-\mu} \hat{P}\left(x t^{-\mu}\right)$ where $\hat{P}$ was determined in in $^{33,29}$

One finds:

$$
F[y, \lambda]=(1-\lambda)^{-\mu} F\left[\frac{1-\lambda}{\lambda}\right] \hat{P}\left[y(1-\lambda)^{-\mu}\right]
$$

The response function can also be computed. Using the formula (A10) of the Appendix A one sees that the matrix $B=H$ and thus:

$$
R\left(t, t^{\prime}\right)=\frac{1}{2} \frac{d<x(t)>}{d t}
$$

and is thus independent of $t^{\prime}$. In fact any response function can be obtained that way. For any observable depending only on time $t$ :

$$
\frac{\delta<O(t)>_{h}}{\delta h_{t^{\prime}}}=\frac{1}{2} \frac{d<O(t)>}{d t}
$$

which comes from the directed nature of the model.

\section{GENERAL DISCUSSION AND BARRIER MODELS}

In this Section we first discuss aging and diffusion properties in low dimensional phase space in terms of scaling of barriers. To illustrate how these ideas work in practice, we study some cases which can be solved analytically, such as the directed model of the last Section with more general wide distributions. These ideas can in principle be applied to study aging in a wider class of models.

\section{A. aging, diffusion and scaling of barriers}

One way to formulate the question of aging is to ask what is the typical size of the next large barrier typically encountered by the particle after time $t_{w}$ (i.e at times $t>t_{w}$ ). The idea is that small barriers have already thermalized. One can usually consider that all barriers of size $T \ln t_{w}-T C$ have already thermalized, where $C$ is a constant which can be chosen large but can be kept fixed as $t_{w} \rightarrow \infty$. (this will be true in 'reasonable' landscapes with fast enough growing barriers, e.g such that diffusion times are smaller than equilibrium times). Thus one must look at the next barrier encountered after $t_{w}$ which has a size bigger than $E_{b}>T \ln t_{w}-C$. For a relaxation behaviour of the type $t / t_{w}$ to occur one must have that a typical particle in a typical environment (i.e that there is a finite fraction of particles and environments) must overcome a barrier within the range $T \ln t_{w}-T C<E_{b}<T \ln t_{w}+T C$ after time $t_{w}$. This is illustrated in Fig. 15. If typically there is no such barrier, e.g the next encountered barrier is always larger, then the particle is typically thermalized for $t / t_{w}=c$ and thus one expects:

$$
\lim _{t_{w} \rightarrow \infty} \overline{Q\left(z, c t_{w}, t_{w}\right)}=\overline{Q_{e q}(z, \tau=\infty)}
$$

which seems to hold for the Sinai model from our simulation results (see section II). This situation is illustrated in Fig. 16 . 
On the other hand, if the particles typically encounter such barriers, then one can have a scaling as $t / t_{w}$. For that one needs an appropriate degeneracy of the barriers. This is the case for the directed model which has en exponential distribution of barriers $e^{-a E}$. At $t_{w}$ there is a finite probability $\sim e^{-a\left(E_{b}-T \ln t_{w}\right)}$ that the next barrier is $E_{b}$. A more precise analysis is presented in the next section.

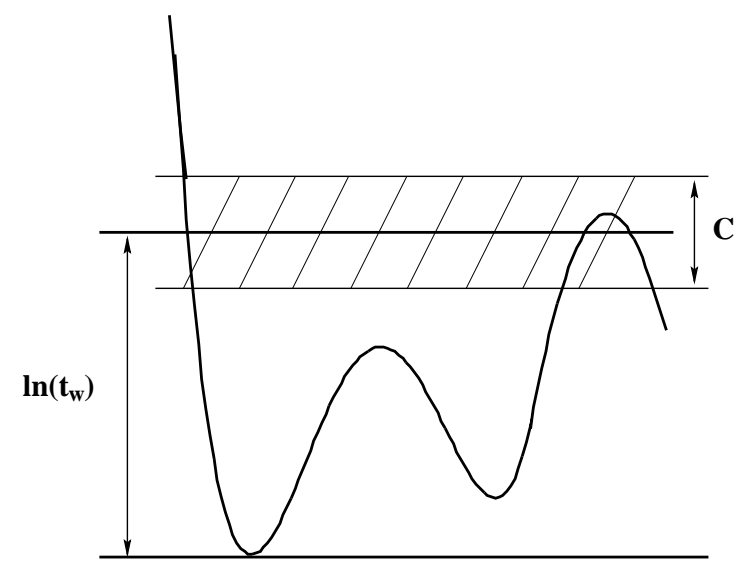

FIG. 15. Barrier condition which leads to aging as $t / t_{w}$

To have aging in $t / t_{w}$ there is of course no need to have a scenario a la Feigelman-Vinokur and also no need for randomness. In the next section we construct a simple model which exhibits a variety of aging and diffusion regimes, but which satisfies the above mentionned property.

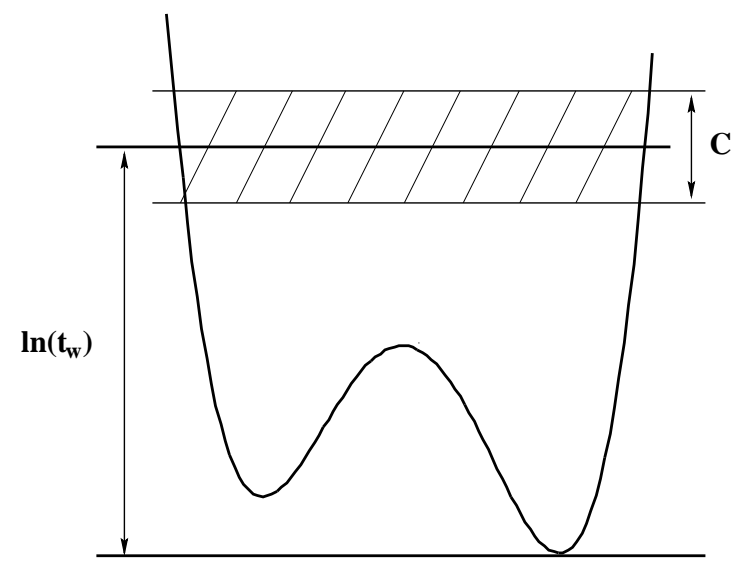

FIG. 16. Barrier in Sinai's model

The property (62) does not mean that there is no aging, but rather that it is degenerate in $t / t_{w}$. In fact (62) suggest that there must be a scaling which gives some aging. One can always define a function $t=g_{c}\left(t_{w}\right)$ such that:

$$
\overline{Q\left(z=0, g_{c}\left(t_{w}\right), t_{w}\right)}=c \quad 0<c<\overline{Q_{e q}(z=0, \tau=\infty)}
$$

The question is how does the function $g_{c}\left(t_{w}\right)$ behaves for large $t_{w}$. A natural possibility is that $g_{c}\left(t_{w}\right) \sim t_{w}^{\mu(c)}$ and indeed we now argue that this is the case in Sinai's model. Note that more generally one can define for each $z$ a function $g\left(c, z, t_{w}\right)$ such that $\overline{Q\left(z=0, g\left(c, z, t_{w}\right), t_{w}\right)}=c$. If the large $t_{w}$ behaviour of this function is the same for all $z$ then one has an aging regime with a non trivial $z$ dependence as in the models of Section $(\mathrm{V})$.

In the symmetric Sinai model the question of the next large barrier to be encountered is difficult to answer analytically but the simple following arguments can be made. Typically at time $t_{w}$ the particle is within a valley (see figure 16). Let us call $U_{\min }$ the lowest energy point of the valley. The walls of the valley are at least $U_{\min }+T \ln t_{w}-T C$. The particle sits at a point which is within $U<U_{\min }+T C$ in energy (since the valley is typically thermalized, sitting 
any higher has a vanishingly small probability).

Geometrical construction of possible such valleys in the energy random-walk landscape of Sinai's model shows that typically the next barrier, i.e the size of the smallest wall is of the order of $(1+a) T \ln t_{w}$ where $a$ is a fluctuating positive random variable. This can be seen from the fractal nature of the landscape. This suggests that a dependence of type $\ln t / \ln t_{w}$, i.e:

$$
\lim _{t, t_{w} \rightarrow \infty} \overline{Q\left(0, t, t_{w}\right)}=F\left[\frac{\ln t}{\ln t_{w}}\right]
$$

which is indeed observed. Note that one also expects, from the argument after (63) that an aging scaling form should exist for any finite $z$ :

$$
\lim _{t, t_{w} \rightarrow \infty} \overline{Q\left(z, t, t_{w}\right)}=F\left[z, \frac{\ln t}{\ln t_{w}}\right]
$$

Thus to predict aging properties one needs to know the statistical properties of sequence of barriers effectively encountered by a particle. It would be appealing to relate it to the geometry of the environment. A first step would be to define in a one dimensional landscape the sequence of next largest barrier encountered starting from an initial point. Let us call them $G_{n}=E_{b}(n)$. Two examples of landscapes are shown in Fig. 17 and Fig. 18. Fig. 17 represents e.g the directed model of last section, with an arbitrary distribution $P(E)$ of barriers (of height $E_{i}$ corresponding to waiting times $\tau_{i}=\exp \left(-E_{i} / T\right)$ ) which generalizes the algebraic waiting times distribution. The important question is how does the series $G_{n}=E_{b}(n)$ grow typically with $n$. This will already give a rough idea of the type of aging one can expect. In particular it may determine the aging scaling function $h(t)$. In Sinai model the series follows a (random) geometric progression $E_{b}(n) \sim \exp (c n)$ as illustrated in Fig. 18 which leads to the observed behaviour (64). In order to have a $t / t_{w}$ behaviour one needs that $E_{b}(n)$ grows typically as $c n$ which is what happens in the directed model. Note that the progression of barriers $E_{b}(n)$ with $n$ depends only on the topology of the lansdcape and for instance of distances along $x$ (different landscapes can have the same $E_{b}(n)$ ).

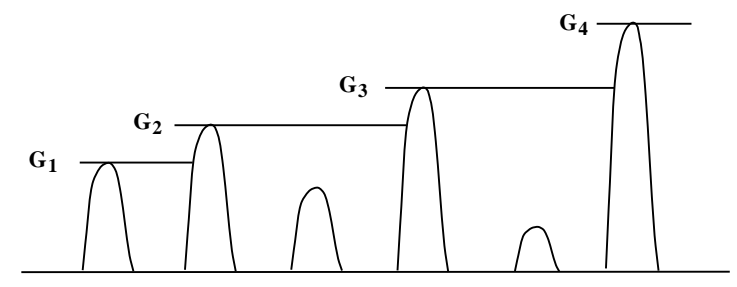

FIG. 17. Sequence of next largest barrier in simple models (see text)

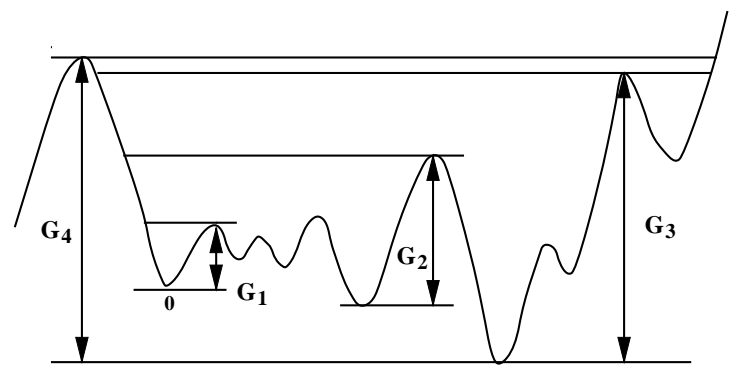

FIG. 18. Sequence of next largest barrier in Sinai's model starting form point 0

Of course to predict more detailed properties one may need to know more: i.e the sequence of barriers effectively encountered by the particle. For this one needs also to know about the well depths. Let us consider e.g. a non directed model but with a landscape as in Fig. 17 where all the wells have the same depth. There the packet will thermalize in a large region (bounded by the next largest barriers) of size $L(t)$. This will result in a geometrical prefactor e.g $Q\left(z=0, t, t^{\prime}\right) \sim L(t)^{-1} F\left[h(t) / h\left(t^{\prime}\right)\right]$. If the well depths grow sufficiently fast then the packet will be concentrated at the bottom and then one may expect a reduced, or even finite prefactor $L(t)=c s t$ (as in Sinai's model). 
Thus if one knows the statistical properties of the sequence of next largest barrier one knows a lot about the aging form. In fact if these barriers grow sufficiently fast with $n$ (faster than $n$ ) we expect that this information is enough to determine these aging form entirely (provided the wells grow also fast enough - see remarks of previous section). This suggests, e.g in Sinai's model, a program to study aging exactly (though it is technically difficult). Let us illustrate these consideration on models where this construction can be done easily.

\section{classification of aging forms and constraints from the CDK bounds}

Before we do so let us present some general considerations about the aging functions $h(t)$. Remember than there is some gauge freedom in choosing them since aging quantities are determined by a fixed ratio $h(t) / h\left(t_{w}\right)=c$. Thus e.g the choice $h(t) \sim t$ is as good as $h(t)=t^{n}$ (with the proper change in $c$ ).

An obvious classification is to distinguish between three classes of functions $h(t)$.

(i) $h(t)$ grows slower than $\mathrm{t}$ (or any power of $t$ ). This corresponds to fast growing barriers. The condition $h(t) / h\left(t_{w}\right)=c$ can also be expressed as $\tau=t-t_{w} \sim t \sim H\left(c, t_{w}\right)$. Examples are:

$$
\begin{aligned}
& h(t) \sim \ln t \quad t \sim \tau \sim t_{w}^{c} \quad(c>1) \\
& h(t) \sim e^{(\ln t)^{a}} \quad t \sim \tau \sim t_{w} \exp \left(\frac{\ln c}{a}\left(\ln t_{w}\right)^{1-a}\right) \quad 0<a<1 \\
& h(t) \sim e^{(\ln t) /(\ln \ln t) \quad t \sim \tau \sim t_{w}\left(\ln t_{w}\right)^{\ln c}}
\end{aligned}
$$

(ii) simplest aging behaviour $h(t)=t$.

(iii) $h(t)$ grows faster than $\mathrm{t}$ (subaging). Then one must have $\tau=t-t_{w} \ll t_{w}$, such as in the following examples:

$$
\begin{aligned}
& h(t) \sim e^{(\ln t)^{a}} \quad \tau=\frac{\ln c}{a} \frac{t_{w}}{\left(\ln t_{w}\right)^{a-1}} \quad a>1 \\
& h(t) \sim e^{t^{a}} \quad \tau \sim \frac{\ln c}{a} t_{w}^{1-a} \quad 0<a<1
\end{aligned}
$$

The CDK bounds discussed in Section II also put general constraints on possible aging forms assuming they have, as in mean field, a non trivial $0<X \neq 1<\infty$ (see Appendix B for definitions). Assume indeed that as in mean field all correlation functions and $X$ are functions of $h\left(t^{\prime}\right) / h(t)$. Then bound (32) implies that:

$$
\frac{d \ln h\left(t^{\prime}\right)}{d t^{\prime}} \ll\left|\frac{d \bar{H}\left(t^{\prime}\right)}{d t^{\prime}}\right|^{1 / 2}
$$

Thus if $\bar{H}\left(t^{\prime}\right) \sim t^{\prime-\alpha}$ one has that

$$
h(t) \ll \exp \left(t^{(1-\alpha) / 2}\right)
$$

which excludes a large class of subaging behaviours (Sinai corresponds to $\alpha=0$ ).

\section{B. sequence of largest barriers}

It is simple in some cases to determine exactly the distribution of the sequence of next largest barriers.

Let us look again at the directed model defined by a set of successive barriers $E_{i}$, identically distributed with a distribution $P(E)$. We denote $H(E)=\int_{E}^{+\infty} P\left(E^{\prime}\right) d E^{\prime}=\operatorname{Proba}\left(E^{\prime}>E\right)$

Let us estimate the probability density that the sequence of successive next largest barriers (see Fig. 17) be $E_{0}, G_{1}, G_{2}, . . G_{n}$. It is by definition:

$$
\begin{aligned}
& Q\left(E_{0}, G_{1}, . . G_{n}\right)=\sum_{k_{1}=0, \infty} . . \sum_{k_{n}=0, \infty} \operatorname{Proba}\left(E_{1}<E_{0}, . . E_{k_{1}}<E_{0}, E_{k_{1}+1}=G_{2}>E_{0}, E_{k_{1}+2}<G_{1}, . . E_{k_{2}+1}=G_{2}>G_{1}, \ldots\right. \\
& \left.. . E_{k_{n}+1}=G_{n}>G_{n-1}\right)
\end{aligned}
$$

This yields immediately:

$$
Q\left(E_{0}, . . G_{n}\right)=p\left(E_{0}\right) \frac{p\left(G_{1}\right) \theta\left(G_{1}-E_{0}\right)}{H\left(E_{0}\right)} \frac{p\left(G_{2}\right) \theta\left(G_{2}-G_{1}\right)}{H\left(G_{1}\right)} \ldots \frac{p\left(G_{n}\right) \theta\left(G_{n}-G_{n-1}\right)}{H\left(G_{n-1}\right)}
$$


It is easy to see that the exponential distribution $p(E)=\exp (-E) \theta(E)$ has a special property. Indeed, in that case:

$$
Q\left(E_{0}, . . G_{n}\right)=\theta\left(E_{0}\right) \theta\left(G_{1}-E_{0}\right) \theta\left(G_{2}-G_{1}\right) . . \theta\left(G_{n}-G_{n-1}\right) e^{-G_{n}}
$$

but this can be written as the product:

$$
Q\left(E_{0}, . . G_{n}\right)=\prod_{i=0,1, . . n} p\left(G_{i}-G_{i-1}\right)
$$

with $G_{0}=E_{0}$ and $G_{-1}=0$. This shows that the difference between successive largest barriers have the same exponential distribution $p(E)$ and are independently distributed. Thus:

$$
G_{n}=\sum_{i=0, n} w_{i}
$$

The central limit theorem can then be used and leads to a Gaussian distribution for the variable $\left(G_{n}-n\right) / \sqrt{n}$. This remarkable property of the exponential distribution allows to understand the Feigelman Vinokur scenario for aging as a linear (random) growth of the next largest barrier $E_{b}(n)=G_{n} \sim c n$

This remarkable property of the exponential distribution also allows to obtain the general solution of the problem of finding the probability of a sequence of largest barrier. Defining $\Phi(E)=-\ln \int_{E}^{+\infty} d E^{\prime} P\left(E^{\prime}\right)$ one has:

$$
P(E) d E=d e^{-\Phi(E)} \theta(E)=\Phi^{\prime}(E) e^{-\Phi(E)} \theta(E) d E
$$

and thus (76) can be put in the form:

$$
\begin{gathered}
Q\left(G_{0}, . . G_{n}\right) d G_{0} . . d G_{n}= \\
d \Phi\left(G_{0}\right) \ldots d \Phi\left(G_{n}\right) \theta\left(G_{0}\right) \theta\left(G_{1}-G_{0}\right) \theta\left(G_{2}-G_{1}\right) . . \theta\left(G_{n}-G_{n-1}\right) e^{-\left(\left(G_{n}-G_{n-1}\right)+\left(G_{n-1}-G_{n-2}\right)+. .+\left(G_{1}-G_{0}\right)+G_{0}\right)}
\end{gathered}
$$

and the sequence of variables $\Phi\left(G_{0}\right), . . \Phi\left(G_{n}\right)$ can be constructed as a "random walk":

$$
\Phi\left(G_{n}\right)=\sum_{i=0}^{n} w_{i}
$$

where the $w_{i}$ are a set of independent variables, identically distributed with an exponential distribution $P(w) d w=$ $e^{-w} \theta(w) d w$. There are several consequences. First the distribution of each $\Phi\left(G_{n}\right)$ is thus a Poisson process:

$$
Q\left(G_{n}\right) d \Phi\left(G_{n}\right)=\frac{\Phi\left(G_{n}\right)^{n}}{n !} e^{-\Phi\left(G_{n}\right)}
$$

and one has the central limit theorem for large $n$ :

$$
\frac{\Phi\left(G_{n}\right)-n}{\sqrt{n}} \rightarrow v
$$

where $v$ is a centered gaussian random variable of unit variance.

One can apply these results to various cases:

(i) algebraically growing barriers:

$P(E) d E=a E^{a-1} e^{-E^{a}} \theta(E) d E$ which corresponds to $\Phi(E)=E^{a}$. Then one has:

$$
G_{n} \sim n^{1 / a}
$$

Note that the randomness (disorder) is only a subleading correction.

(ii) exponentially growing barriers:

$P(E) d E=(1+E)^{-(a+1)} \theta(E) d E$ which corresponds to $\Phi(E)=a \ln E$. Then one has:

$$
G_{n} \sim e^{n / a}
$$

The variable $G_{n}$ has a log normal distribution. 


\section{C. consequences: aging properties}

Having determined exactly the sequence of next largest barriers one can construct a toy model which will mimic the exact diffusion process. We claim that if barriers grow fast enough (faster than $E_{n} \sim n$ ) it will give the exact aging behaviour. It can be applied to a variety of landscape, but let us apply it here only to the previously considered directed model.

The toy model amounts to approximate the quantity $\overline{Q\left(z, \tau, t_{w}\right)}$ at $z=0$ (i.e the probability to remain still between $t_{w}$ and $\left.t_{w}+\tau\right)$ as:

$$
\overline{Q\left(\tau, t_{w}\right)} \equiv \overline{Q\left(0, t_{w}+\tau, t_{w}\right)}=\sum_{k=0}^{+\infty} \theta\left(G_{k-1}<T \ln t_{w}<G_{k}\right) \exp \left(-\tau e^{-G_{k} / T}\right)
$$

This means that at $t_{w}$ all barriers smaller than $T \ln t_{w}$ have been overcome and that the only relaxation process is to go over the next highest barrier. This is illustrated in Fig. 17 which is adequate for the directed model. It thus supposes that the probability to be in another well is zero, which is correct for the directed model if barriers grow fast enough (which means distribution of waiting times wider than power laws)

This can be rewritten as:

$$
Q\left(\tau, t_{w}\right)=\sum_{k=0}^{+\infty}\left(\theta\left(\Phi_{w}-\Phi_{k-1}\right)-\theta\left(\Phi_{w}-\Phi_{k}\right)\right) \exp \left(-\tau e^{-\frac{1}{T} \Phi^{-1}\left(\Phi_{k}\right)}\right)
$$

where $\Phi_{k}=\Phi\left(G_{k}\right)$ and $\Phi_{w}=\Phi\left(T \ln t_{w}\right)$. Because of the above statistical properties of the sequence this yields upon averaging:

$$
\overline{Q\left(\tau, t_{w}\right)}=\int d \phi \sum_{k=0}^{+\infty} Q_{k}(\phi) \int_{0}^{+\infty} d w e^{-w}\left(\theta\left(\Phi_{w}-\phi+w\right)-\theta\left(\Phi_{w}-\phi\right)\right) \exp \left(-\tau e^{-\frac{1}{T} \Phi^{-1}(\phi)}\right)
$$

and we can use (84) namely that $\sum_{k=0}^{+\infty} Q_{k}(\phi)=1$. In the large time limit one can shift the integrand globally by $\phi \rightarrow \phi+\Phi_{w}$ without edge effects (though one must be careful not to shift terms independently because of divergent integrals). Thus:

$$
\overline{Q\left(\tau, t_{w}\right)}=\int d \phi \int_{0}^{+\infty} d w e^{-w}(\theta(-\phi+w)-\theta(-\phi)) \exp \left(-\tau e^{-\frac{1}{T} \Phi^{-1}\left(\phi+\Phi_{w}\right)}\right)
$$

This can be rewritten, after integration by parts as:

$$
\overline{Q\left(\tau, t_{w}\right)}=\int_{0}^{+\infty} d v e^{-v} \exp \left(-\tau e^{-\frac{1}{T} \Phi^{-1}\left(v+\Phi_{w}\right)}\right)
$$

This is our general result for this toy model.

Let us estimate (92) first for models with fast enough growing barriers, i.e faster than $\Phi(E) \sim E$. We introduce the aging scaling function $h(t)=e^{\Phi(T \ln t)}$ and thus $\Phi(x)=\ln h\left(e^{x / T}\right)$ and $\Phi^{-1}(y)=T \ln h^{-1}\left(e^{y}\right)$. One can rewrite:

$$
\overline{Q\left(\tau, t_{w}\right)}=\int_{0}^{+\infty} d w e^{-w} \exp \left(-e^{-\frac{1}{T} S\left(w, \tau, t_{w}\right)}\right)
$$

with $S\left(w, \tau, t_{w}\right)=\Phi^{-1}\left(w+\Phi\left(T \ln t_{w}\right)\right)-T \ln \tau$. It turns out that if barriers grow fast enough the function $\exp \left(-e^{-S}\right)$ acts exactly as a theta function $\theta(S)$ and the result is simply:

$$
\overline{Q\left(\tau=t-t_{w}, t_{w}\right)}=\frac{h\left(t_{w}\right)}{h(\tau)} \quad\left(\tau \gg t_{w}\right)
$$

This can be shown by a careful examination of the asymptotics. The point is that if one takes $\tau$ and $t_{w}$ to $+\infty$ such that the ratio $h\left(t_{w}\right) / h(\tau)$ is fixed then the result is $(94)$. Since barriers grow fast enough one has lim $h\left(t_{w}\right) / h(t)=$ $\lim h\left(t_{w}\right) / h(\tau)$. A more correct version of the above statement is that:

$$
\lim _{t_{w} \rightarrow \infty, t \rightarrow \infty, \frac{h\left(t_{w}\right)}{h(t)}=y} \overline{Q\left(\tau=t-t_{w}, t_{w}\right)}=y
$$


It is interesting to note that one has exactly:

$$
e^{w}=\frac{h(t)}{h\left(t_{w}\right)}
$$

and thus the above result follows from the exponential distribution of $w$. Note that this is like taking the $T=0$ limit and thus if barriers grow fast enough we are dealing with a $T=0$ fixed point. Note that our result can also be rewritten quite generally as:

$$
\overline{Q\left(\tau=t-t_{w}, t_{w}\right)}=\frac{\int_{t}^{+\infty} P(\tau) d \tau}{\int_{t_{w}}^{+\infty} P(\tau) d \tau}
$$

as a function of the waiting time distribution $\tau=e^{E / T}$. In particular we have determined that the aging function is exactly:

$$
h(t)=\frac{1}{\int_{t}^{+\infty} P(\tau) d \tau}
$$

One can also define the probability that at time $t_{w}$ the particle is next to a barrier (i.e in a site) of waiting time $\tilde{\tau}$, i.e:

$$
\overline{Q\left(\tau, t_{w}\right)}=\int_{0}^{+\infty} d \tilde{\tau} P_{t_{w}}(\tilde{\tau}) e^{-\tau / \tilde{\tau}}
$$

Then the above formulae yields that this "aged" waiting time distribution is:

$$
P_{t_{w}}(\tilde{\tau}) d \tilde{\tau}=\frac{h\left(t_{w}\right)}{h(\tilde{\tau})} \frac{d h(\tilde{\tau})}{h(\tilde{\tau})} \theta\left(h(\tilde{\tau})-h\left(t_{w}\right)\right)
$$

This also takes the simple form:

$$
P_{t_{w}}(\tilde{\tau}) d \tilde{\tau}=\frac{P(\tilde{\tau}) d \tilde{\tau}}{\int_{t_{w}}^{+\infty} P\left(\tilde{\tau^{\prime}}\right) d \tilde{\tau^{\prime}}} \theta\left(\tilde{\tau}-t_{w}\right)
$$

which we have shown holds exactly.

We can now apply these results it to several cases.

(i) algebraically growing barriers:

$\Phi(E)=E^{a}$ with $a<1$. Then one has $h(t)=\exp \left((T \ln t)^{a}\right)$.

$$
\overline{Q\left(\tau=t-t_{w}, t_{w}\right)}=\exp \left(-\left[(T \ln t)^{a}-\left(T \ln t_{w}\right)^{a}\right]\right)
$$

(ii) exponentially growing barriers:

$P(E) d E=(1+E)^{-(a+1)} \theta(E) d E$ which corresponds to $\Phi(E)=a \ln E$ and $h(t)=(T \ln t)^{a}$. Then one has:

$$
\overline{Q\left(\tau=t-t_{w}, t_{w}\right)}=\left(\frac{\ln t_{w}}{\ln t}\right)^{a}
$$

Let us now apply the above model in the case of linearly growing barriers $(\Phi(E)=E)$. There we know that the assumption that the whole packet is concentrated nest to the next highest barrier is certainly not exact. Indeed since there are many barriers within $T \ln t_{w}-C<E<T \ln t_{w}+C$ the packet will be spread out (barrier degeneracy). It is instructive though, to see how well this toy model does in that case.

Setting $y=\tau / t_{w}$ one gets:

$$
\overline{Q(y)}=\int_{0}^{+\infty} d w e^{-w} \int_{0}^{w} d \phi \exp \left(-y e^{-\phi / T}\right)
$$

After integration by part and change of variables: 


$$
\overline{Q(y)}=\int_{0}^{+\infty} d w e^{-w} \exp \left(-y e^{-w / T}\right)=T y^{-T} \int_{0}^{y} \frac{d \lambda}{\lambda} \lambda^{T} e^{-\lambda}
$$

For large $y$ it behaves as:

$$
\overline{Q(y)} \sim T \Gamma[T] y^{-T}
$$

which is to be compared with (53) ( $T$ is exactly $\mu, T=\mu$, from the relation between waiting times and barrier heights). The toy model gives exactly the exponent, but not the prefactor. We note however that at small $T$ the prefactor becomes exact which is in agreement with the fact that for faster growing barriers the toy model becomes exact. At small $y$ the toy model however does not yield the non analyticity at $\tau<<t_{w}$, which thus entirely originates from the initial (fractal) dispersion of the packet at $t_{w}$ over several wells.

This confirms our physical picture: only maxima start playing a role when barriers $G_{n}$ grow faster than $n$. The case $G_{n} \sim n$ is the marginal case when finer information is important.

Note that the exact result (51) can be put under the form:

$$
\begin{aligned}
& \overline{Q\left(\tau, t_{w}\right)}=\int_{0}^{+\infty} d u \phi(u) e^{-u \tau / t_{w}} \\
& \phi(u)=\frac{\sin (\pi T)}{\pi} \frac{e^{-u}}{u} \int_{0}^{u} \frac{d t}{t \Gamma[T]} t^{T} e^{t}
\end{aligned}
$$

to be compared with the toy model which has a $\phi(u)=T u^{T-1} \theta(0<u<1)$. Thus one can see that for large $w$ the $e^{-w}$ is correct, however the barriers which are not large, or the one at $w<0$ (smaller than $t_{w}$ ) start playing an important role.

The role of smaller barriers can be illustrated as follows. The correct distribution of the waiting time $\tilde{\tau}$ of the site where the particle is at $t_{w}$ is computed in the Appendix. It reads:

$$
P_{t_{w}}(\tilde{\tau}) d \tilde{\tau}=\frac{\sin (\pi \mu)}{\pi} \frac{d \tilde{\tau}}{t_{w}}\left(\frac{t_{w}}{\tilde{\tau}}\right)^{1+\mu} \int_{0}^{1} e^{-\frac{t_{w}}{\tau}(1-u)} \frac{u^{\mu-1}}{\Gamma[\mu]}
$$

This should be compared with what the barrier model which gives:

$$
P_{t_{w}}(\tilde{\tau}) d \tau=\mu \frac{d \tilde{\tau}}{t_{w}}\left(\frac{t_{w}}{\tilde{\tau}}\right)^{1+\mu} \theta\left(\tilde{\tau}-t_{w}\right)
$$

from the above fomulae (102) and the identification $\tilde{\tau}=e^{w / T}$. As above, the toy model gives the correct power law dependence for large waiting times (the prefactor itself becoming exact when $\mu \rightarrow 0$ ). There is however an accumulation of smaller barriers effectively seen by the particle, which is not captured.

Let us conclude this section by noting that this type of distribution of next largest barriers can be used to analyze a large variety of models. For instance the analysis of the symmetric waiting time model in $d=1$ or of directed model with several branches will be quite similar but goes beyond this paper.

\section{A SOLVABLE MODEL WITH AGING AND DIFFUSION}

We will now present a solvable model which exhibits simultaneously a non trivial aging regime and a non trivial diffusion regime. The third regime (the quasi-equilibrium one) is degenerate (it is reduced to a point). More properly this is really a class of solvable models, and we will only study a few.

In view of the discussion about barriers of the preceding section the best way to construct a one dimensional diffusion model with a non trivial aging regime (i.e such that there is a finite probability that $z=x(t)-x\left(t_{w}\right)$ remains finite when both $t$ and $t_{w}$ are large) is to make sure that the next largest barrier seen after time $t_{w}$ is equal to the previous one plus a constant (for aging as $t / t_{w}$ ). A natural landscape is thus to look at a succession of barriers $E_{b}^{n} \sim n$. However one wants the valleys also to become deeper and deeper, otherwise the thermal packet will be too extended. Thus a natural choice is also to suppose valleys to scale as $E_{\min }^{n} \sim-n$, a landscape represented in Fig. 19 It turns out that the continuous version of this model, as well as some generalizations, can be solved exactly in a very simple way. 


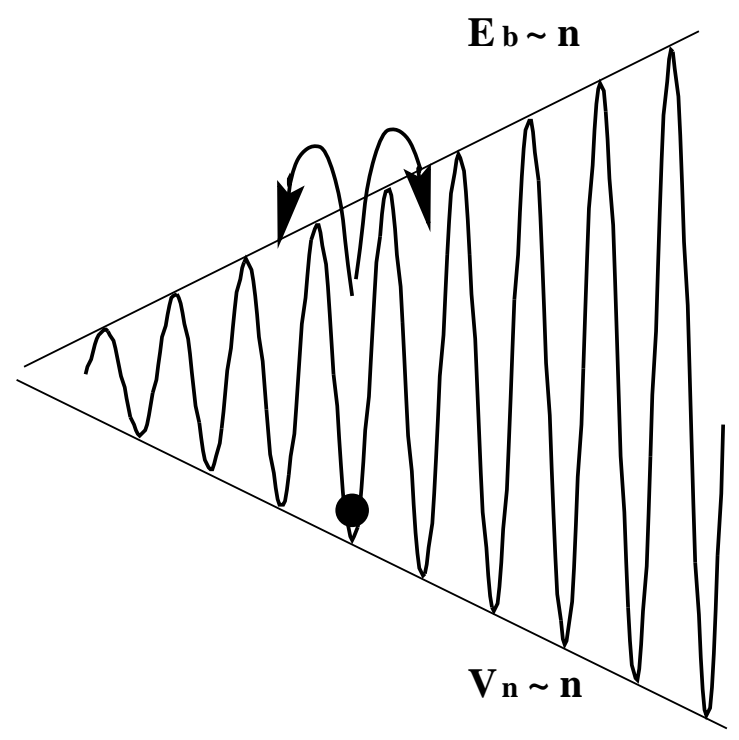

FIG. 19. Aging model studied in the text in the continuum limit

\section{A. the general model and its solution}

Let us consider the following one dimensional diffusion equation:

$$
\partial_{t} P(x, t)=\partial_{x}\left(D(x)\left(\partial_{x} P(x, t)-F(x) P(x, t)\right)\right)
$$

This model corresponds to diffusion in a landscape with both barriers $E_{b}(x)$ and a potential $U(x)$ (valleys) such that:

$$
D(x)=e^{-E_{b}(x)} \quad U^{\prime}(x)=-F(x)
$$

here and in what follows we will set the temperature $T=1$ for convenience but it can be easily put back in. The potential is defined by the fact that the equilibrium zero current measure is $P_{e q}(x)=\exp (-U(x))$. The general model (112) cannot be solved but there is a particular case which can be solved easily, which is:

$$
U(x)=-E_{b}(x) / 2
$$

The model is defined by giving a function $\Phi(x)$ such that:

$$
e^{U(x)}=e^{-E_{b}(x) / 2}=\Phi(x)
$$

Then the diffusion equation becomes:

$$
\partial_{t} P(x, t)=\partial_{x}\left(\Phi(x) \partial_{x}(\Phi(x) P(x, t))\right)
$$

and is easily solved since one can define a new variable $u$ and a new probability $G(u, t)$ such that:

$$
\frac{d u}{d x}=\frac{1}{\Phi(x)} \quad P(x, t) d x=G(u(x), t) d u(x)
$$

In the variable $u$ the system is a free diffusion problem !

$$
\partial_{t} G(u, t)=\partial_{u}^{2} G(u, t)
$$

Clearly for the choice $\Phi(x)=e^{x}$ and thus $E_{b}(x)=2 x$ this model is some continuum limit of the one represented in Fig. 19. It will have the same expected large time properties. Note that the quasi-equilibrium regime will be lost 
in this limit (i.e reduced to a delta function, see below) as the size of each well will become infinitesimal. It would be nice to be able to solve directly the model of Fig.19. to obtain also the small times behaviour. In any case the results presented here for large times will be the same (they are not an artefact of the continuous limit).

One must define carefully the boundary conditions. There are basically two choices that we will study:

free boundary conditions

One can study a barrier landscape $E_{b}(x)$ defined from $-\infty<x<\infty$. This could either be a random landscape $U(x)$ or a deterministic one with growing barriers in $d=1$ which we then should choose symmetric for definiteness for instance $U(x)=-|x|$. Then a natural definition of $u$ is :

$$
u(x)=\int_{0}^{x} d x^{\prime} e^{-U\left(x^{\prime}\right)}
$$

and $-\infty<u<\infty$.

The Green function is thus simply unbounded diffusion

$$
G\left(u, t \mid u_{0}, 0\right)=\frac{1}{\sqrt{4 \pi t}} e^{-\frac{\left(u-u_{0}\right)^{2}}{4 t}}
$$

which yields the Green function for the original equation:

$$
P\left(x, t \mid x_{0}, 0\right)=\frac{1}{\sqrt{4 \pi t}} e^{-U(x)} e^{-\frac{\left(\int_{x_{0}}^{x} d x^{\prime} e^{\left.-U\left(x^{\prime}\right)\right)^{2}}\right.}{4 t}}
$$

We will be interested in the probability of displacements $z$ between $t^{\prime}$ and $t$ :

$$
Q\left(z, t, t^{\prime}\right)=\int d x P\left(x+z, t \mid x, t^{\prime}\right) P\left(x, t^{\prime} \mid x_{0}=0,0\right)
$$

which formally reads:

$$
Q\left(z, t, t^{\prime}\right)=\int d x \frac{1}{4 \pi \sqrt{t^{\prime}\left(t-t^{\prime}\right)}} e^{-U(x)-U(x+z)} e^{-\frac{\left(\int_{0}^{x} d x^{\prime} e^{\left.-U\left(x^{\prime}\right)\right)^{2}}\right.}{4 t^{\prime}}-\frac{\left(\int_{x}^{x+z} d x^{\prime} e^{\left.-U\left(x^{\prime}\right)\right)^{2}}\right.}{4\left(t-t^{\prime}\right)}}
$$

Another convenient form is to write:

$$
Q\left(z, t, t^{\prime}\right)=\int d u_{1} d u_{2} \frac{1}{4 \pi \sqrt{t^{\prime}\left(t-t^{\prime}\right)}} \delta\left(z-\left(x\left(u_{1}+u_{2}\right)-x\left(u_{1}\right)\right)\right) e^{-\frac{u_{1}^{2}}{4 t^{\prime}}-\frac{u_{2}^{2}}{4\left(t-t^{\prime}\right)}}
$$

where $x(u)$ is the function implicitly defined by (119).

reflecting boundary at $u=0$

Since we will be interested in landscapes with growing barriers such as depicted in Fig. 19. it is useful in certain cases to introduce a reflecting boundary on the left. One can choose for definiteness a landscape such that $U(x \rightarrow$ $-\infty) \rightarrow+\infty$, define $u(x)=\int_{-\infty}^{x} d x^{\prime} e^{-U\left(x^{\prime}\right)}$ and use a reflecting boundary at $u=0$. This naturally avoids the particule being either in one half space or the other. For instance for the lanscape $E_{b}(x)=2 x$, for which $u(x)=e^{x}$ the reflecting boundary is at $x=-\infty$. Because of reflecting boundaries one must choose the free propagator with $\partial_{u} G(u=0)=0$, i.e:

$$
G\left(u, t \mid u^{\prime}, t^{\prime}\right)=\theta(u) \frac{1}{\sqrt{4 \pi\left(t-t^{\prime}\right)}}\left(e^{-\frac{\left(u-u^{\prime}\right)^{2}}{4\left(t-t^{\prime}\right)}}+e^{-\frac{\left(u+u^{\prime}\right)^{2}}{4\left(t-t^{\prime}\right)}}\right)
$$

We will be interested in:

$$
Q\left(z, t, t^{\prime}\right)=\int d x P\left(x+z, t \mid x, t^{\prime}\right) P\left(x, t^{\prime} \mid x_{0}=-\infty, 0\right)
$$

where we have chosen for convenience the initial condition at $x_{0}=-\infty$ (and thus $u_{0}=0$ ). This is a purely technical point on the definition of the model and has no bearing on the physics. Indeed since we have chosen $E_{b}(x \rightarrow-\infty) \rightarrow-\infty$ and the initial condition will be immaterial since it takes only a finite time in this model to reach finite $x$ values and we are interested only in the later (long time) behaviour. Thus using: 
Using $P\left(x, t \mid x^{\prime}, t^{\prime}\right)=\Phi(x)^{-1} G\left(u(x), t \mid u\left(x^{\prime}\right), t^{\prime}\right)$ one finds:

$$
Q\left(z, t, t^{\prime}\right)=\int d x \frac{1}{4 \pi \sqrt{t^{\prime}\left(t-t^{\prime}\right)}} e^{-U(x)-U(x+z)} e^{-\frac{(u(x))^{2}}{4 t^{\prime}}}\left(e^{-\frac{(u(x+z)-u(x))^{2}}{4\left(t-t^{\prime}\right)}}+e^{-\frac{(u(x+z)+u(x))^{2}}{4\left(t-t^{\prime}\right)}}\right)
$$

Let us conclude this section by indicating that a more general case can be solved (see also D), i.e brought back to:

$$
\partial_{t} G(u, t)=\partial_{u}^{2} G(u, t)-v \partial_{u} G(u, t)
$$

which corresponds to:

$$
U(x)=-\left(E_{b}(x) / 2+v \int_{0}^{x} d y e^{E_{b}(y) / 2}\right)
$$

These models corresponds to either valleys and barriers scaling differently and we will not study these models here. We note that the directed model of section III can be seen as a particular case of the class of models introduced here. Indeed the case (128) corresponds to the diffusion in $x$ given by:

$$
v t^{\prime}+\sqrt{t^{\prime}} w=u(x)-u\left(x_{0}\right)=\int_{x_{0}}^{x} e^{-U\left(x^{\prime}\right)} d x^{\prime}
$$

where $w$ is a normalized gaussian variable. In the fully directed case (large $v$ ) and for a judicious choice of the (random) $U(x)$ one can recover the directed models.

\section{B. solution for linearly growing barriers}

It corresponds to the model of Fig. 15. Again one can either consider the symmetric landscape taking $U(x)=-|x|$ and thus $x(u)=\operatorname{sgn}(u) \ln (1+|u|)$. Or one can take the "half landscape" with a reflexive barrier at $x_{0}=-\infty$ which we will discuss first.

The above formulae give in that case, for the single time packet:

$$
P\left(x, t \mid x_{0}, 0\right)=\frac{2}{\sqrt{4 \pi t}} e^{x} e^{-\frac{e^{2 x}}{4 t}}
$$

The generating function of moments is $\left\langle e^{\lambda x}\right\rangle=\Gamma[(1+\lambda) / 2](4 t)^{\lambda) / 2} / \sqrt{\pi}$. Thus one has:

$$
\begin{aligned}
& <x(t)>=\frac{1}{2} \ln (t / c) \\
& <x^{2}(t)>-<x(t)>^{2}=\pi^{2} / 8
\end{aligned}
$$

where $c=\ln \gamma$ ( $\gamma$ is Euler's constant). In fact the packet has a limit shape as one can see by performing the shift $x=\frac{1}{2} \ln (4 t)+\tilde{x}$. Then the distribution of $\tilde{x}$ is asymptotically time independent:

$$
P(\tilde{x}, t) \sim \frac{2}{\sqrt{\pi}} e^{x} e^{-e^{2 \tilde{x}}}
$$

Thus the packet has a constant width and simply spreads over a few wells (in Fig. 15) with its center moving logarithmically towards the right. Thus, since there is some degeneracy of barriers one expects a $t / t_{w}$ aging behaviour. Indeed one gets for the two time packet:

$$
Q\left(z, t, t^{\prime}\right)=\int_{0}^{+\infty} d u u \frac{e^{z}}{2 \pi \sqrt{t^{\prime}\left(t-t^{\prime}\right)}} e^{-\frac{u^{2}}{4 t^{\prime}}}\left(e^{-\frac{u^{2}\left(e^{z}-1\right)^{2}}{4\left(t-t^{\prime}\right)}}+e^{-\frac{u^{2}\left(e^{z}+1\right)^{2}}{4\left(t-t^{\prime}\right)}}\right)
$$

This yields to an aging form for the distribution of displacements between $t^{\prime}$ and $t$, given by:

$$
Q\left(z, t, t^{\prime}\right)=\frac{e^{z}}{\pi}\left(\frac{\Gamma}{\left(e^{z}-1\right)^{2}+\Gamma^{2}}+\frac{\Gamma}{\left(e^{z}+1\right)^{2}+\Gamma^{2}}\right) \quad \Gamma=\frac{\sqrt{t-t^{\prime}}}{\sqrt{t^{\prime}}}
$$

which, in the variable $w=e^{z}$, is the sum of two Lorentzian of width $\Gamma$ the first one centered around $w=1$ and the second one which is its mirror image (with the mirror at $w=0$ ). This result is natural considering that the ratio 
of two independent gaussian variables with unit variance $v=v_{1} / v_{2}$ is the Lorentzian $P(v)=1 /\left(\pi\left(v^{2}+1\right)\right)$ and that one has:

$$
z=x\left(u_{1}+u_{2}\right)-x\left(u_{1}\right)=\ln \left(1+\frac{u_{2}}{u_{1}}\right)=\ln (1+\Gamma v)
$$

where $u_{1}=\sqrt{t^{\prime}} v_{1}$ is the positive gaussian variable representing the diffusion process on the half line and $u_{2}=\sqrt{\tau} v_{2}$ another gaussian variable representing the later diffusion process, constrained so that the sum $u_{1}+u_{2}$ remains positive, hence the mirror. The total weight in the second packet is $p=(\arctan \Gamma) / \pi$ and in $1-p$ the first.

Thus the first Lorentzian packet in (136) corresponds to particles which have remained in the region $x\left(t^{\prime}\right)$ while the others have crossed to the mirror at $x=-\infty$ at least once and came back to that region. We are mostly interested with this first packet (which always contains a fraction $>1 / 2$ of particles) but the second packet will always be there as a mainly technical feature of the model. If we chose instead the symmetric environment, these would be two separate aging packets one around $x\left(t^{\prime}\right)$ and the other around $-x\left(t^{\prime}\right)$. Aging then occurs only within each packet (there are diffusion events between packets) while here one has aging in all the packet.

Indeed the above distribution (136) clearly exhibits an aging scaling form of the type:

$$
Q\left(z, t, t^{\prime}\right)=\tilde{Q}\left(z, \frac{t}{t^{\prime}}\right)=\tilde{Q}\left(z, \frac{h(t)}{h\left(t^{\prime}\right)}\right)
$$

thus $h(t)=t$ in this model. The function $\tilde{Q}$ indeed depends on the times only through $\Gamma$ which itself can be written as:

$$
\Gamma=f\left[\frac{h(t)}{h\left(t^{\prime}\right)}\right]
$$

where the form of $f(x)$ and $h(t)$ is univoquely determined as $h(t)=t$ and $f(x)=\sqrt{1-x}$. Thus, as in mean field ${ }^{36}$ there is a singularity at the beginning of the aging regime: the $\beta$ exponent is equal to $\beta=1 / 2$.

It is interesting to note though that at the beginning of the aging regime $\Gamma<<1$, there is anomalous behaviour of the moments $z^{n}$, because the Lorentzian has diverging moments:

$$
<z^{n}>=\int_{-1 / \Gamma}^{+\infty}(\ln (1+\Gamma w))^{n} \frac{d w}{1+w^{2}}
$$

One finds in particular $\langle z\rangle=1 / 2 \ln \left(1+\Gamma^{2}\right)=1 / 2 \ln \left(t / t^{\prime}\right)$ exactly.

$$
<(z-<z>)^{n}>=\frac{\Gamma}{2 \pi \sqrt{1+\Gamma^{2}}} \int_{-\infty}^{+\infty} d u u^{n}\left(\frac{1}{\cosh [u]-\frac{1}{\sqrt{1+\Gamma^{2}}}}+\frac{1}{\cosh [u]+\frac{1}{\sqrt{1+\Gamma^{2}}}}\right)
$$

One finds that for $n>1<(z-<z>)^{n}>\sim \Gamma \Gamma[n+1]$ (i.e $P(u)=\Gamma e^{-u}$ ) which is strong intermittence.

For widely separated time scales there is also a diffusion regime in this model. At large $t$ one gets:

$$
z=\ln (v)+\ln (t)-\ln \left(t^{\prime}\right)
$$

where $v$ has a Lorentzian distribution. Thus the diffusing packet has a finite size (note that since for a Lorentzian $<\ln (v)>=0$ (inversion symmetry) one recovers the above result). Thus there is a diffusion regime defined by $z, t, t^{\prime} \rightarrow \infty$ with $z / \ln t$ and $\ln t / \ln t^{\prime}$ fixed. It has no thermal fluctuations (it is completely determinist) and the diffusion scaling function reads:

$$
Q\left(z, t, t^{\prime}\right) d z \sim \frac{d z}{\ln t} \delta\left(\frac{z}{\ln t}-\left(1-\frac{\ln (t)}{\ln \left(t^{\prime}\right)}\right)\right)
$$

As in SR mean field models the aging regime smoothly merges in the diffusion regime ${ }^{36}$

This model thus contains both an aging regime in $t / t_{w}$ and a diffusion regime with a different scaling. Only the FDT regime cannot be seen, since it has disappeared in the continuum limit.

Finally note that this model seems to violate the quasistatic assumption. Indeed in a box of finite size $L$, or with periodic boundary conditions and taking $t_{w} \rightarrow \infty$, the packet will converge towards the equilibrium measure. Since $U(x)=-x$ this will result in an equilibrium packet of finite size. However the dynamical regime $t-t^{\prime} \ll t^{\prime}$ leads only to a delta funtion packet at $\delta(z)$, thus totally different from the equilibrium one. We expect this feature to persist for the model of Fig. 15, i.e it is not an artefact of the continuous limit ! 
Other quantities can be computed in this model. Let us give some examples:

calculation of separation of replicas

One can also compute the separation of two thermal replicas which are allowed to split at time $t_{w}$. This quantity was studied in ${ }^{10}$.

One has:

$$
\begin{aligned}
& Q_{2}\left(z, t_{w}, \tau\right)=\int d y d x P\left(y, t \mid x, t_{w}\right) P\left(y+z, t \mid x, t_{w}\right) P\left(x, t_{w} \mid x_{0}=-\infty, 0\right) \\
& =e^{z} \int_{0}^{+\infty} d u_{1} \int_{0}^{+\infty} d u u_{1} G\left(u_{1}, \tau \mid u\right) G\left(u_{1} e^{z}, \tau \mid u\right) G\left(u, t_{w} \mid 0\right) \\
& G\left(u, \tau \mid u^{\prime}\right)=\frac{1}{\sqrt{4 \pi \tau}}\left(\exp \left(-\frac{\left(u-u^{\prime}\right)^{2}}{4 \tau}\right)+\exp \left(-\frac{\left(u+u^{\prime}\right)^{2}}{4 \tau}\right)\right)
\end{aligned}
$$

and one finds:

$$
\begin{aligned}
& Q_{2}\left(z, t_{w}, \tau\right)=\frac{e^{z}}{\pi}\left(\frac{\Gamma}{\left(e^{z}-m\right)^{2}+\Gamma^{2}}+\frac{\Gamma}{\left(e^{z}+m\right)^{2}+\Gamma^{2}}\right) \\
& \Gamma=\sqrt{\frac{\tau\left(2 t_{w}+\tau\right)}{\left(t_{w}+\tau\right)^{2}}} \quad m=\frac{t_{w}}{t_{w}+\tau}
\end{aligned}
$$

with $\tau=t-t^{\prime}$. Note that this distribution is symmetric under $z \rightarrow-z$ because of the relation $\Gamma^{2}+m^{2}=1$. For large $\tau \gg t_{w}$ one finds that the distribution of $x=e^{z}$ goes to a fixed half Cauchy unit $\operatorname{distribution} \theta(x) 1 /\left(\pi\left(1+x^{2}\right)\right)$. Thus for large time we find that the two replicas evolve within a finite distance, but this distance is larger than the dynamical $q_{E A}$ which is zero for this problem.

Note that when $\tau / t_{w} \ll 1$ one recovers exactly the previous $Q\left(z, t, t^{\prime}\right)$.

non trivial FDT violation ratio?

It is interesting to know if one can find finite dimensional models with a non trivial FDT violation ratio $X$ as in mean field.

Here one can also compute the response to an additional field, i.e the remanent magnetization decay. The calculation is indicated in the Appendix E. Though it does appear that in some sense this model has a non trivial FDT violation ratio $X$ similar to mean field we were not able to exhibit it in a clear way. If one looks at the finite fraction of the packet which has not touched the reflecting boundary, it has clearly a non trivial $X$. But on any global quantity we have looked at the boundary effects always introduce a cutoff which changes the expected result. We do not know if this is a purely technical limitation, and if the model can be improved to really exhibit a non trivial and properly

defined $X$ or if this is a more fundamental limitation. We still present some of the calculations in the Appendix E for the brave who is encourage to improve on it.

\section{C. solution of more general deterministic model}

\section{1. barriers growing faster than linear}

One can study cases where $E_{b}(x) \sim|x|^{b}$ with $b>1$. From the Section IV we expect that aging should have simple properties. For the "half landscape" model, using $u(x) \sim \exp \left(x^{b}\right)$ for $x>0$ and a left reflecting wall at $u=1$, one finds an aging regime with no thermal fluctuation:

$$
\begin{aligned}
& z=\left(\ln \left(\sqrt{t^{\prime}} v_{1}+\sqrt{t-t^{\prime}} v_{2}\right)^{1 / b}-\left(\ln \left(\sqrt{t^{\prime}} v_{1}\right)\right)^{1 / b}\right. \\
& z=(\ln \sqrt{t})^{1 / b}-\left(\ln \sqrt{t^{\prime}}\right)^{1 / b}
\end{aligned}
$$

where $v_{1}$ and $v_{2}$ are uncorrelated normalized gaussian variables. We have performed an expansion, in the regime of interest $t \gg t^{\prime}$ e.g:

$$
\left(\ln \left(\sqrt{t^{\prime}} v_{1}\right)\right)^{1 / b} \sim\left(\ln \sqrt{t^{\prime}}\right)^{1 / b}+\frac{\ln \left(v_{1}\right)}{\left(\ln \sqrt{t^{\prime}}\right)^{1-1 / b}}
$$


and dropped the contributions of the noise parts $v_{1}$ and $v_{2}$ which vanish in the limit of large $t$ and $t^{\prime}$ since $b>1$. Thus aging becomes thermally deterministic as:

$$
z=\ln \frac{h(t)}{h\left(t^{\prime}\right)}
$$

with $h(t)=\exp \left(C(\ln t)^{1 / b}\right)$ and $C=(1 / 2)^{1 / b}$. These results are strikingly similar with the result (96) of the previous Section. In fact the result (152) is also similar to what was found in mean field in ${ }^{36}$.

There is also a diffusion regime, which is identical to the end of the aging one (it merges smoothly into it). Indeed:

$$
z=(\ln \sqrt{t})^{1 / b}\left(1-\left(\frac{\ln t^{\prime}}{\ln t}\right)^{1 / b}\right.
$$

\section{2. barriers growing slower than linear (subaging)}

Similarly, in the subaging case where $E_{b}(x) \sim x^{b}$ with $b<1$ one finds:

$$
z=\ln \left(1+\sqrt{\tau} v_{2}+\sqrt{t^{\prime}} v_{1}\right)^{1 / b}-\ln \left(1+\sqrt{t^{\prime}} v_{1}\right)^{1 / b}
$$

Performing expansions it gives:

$$
z=\frac{1}{b} \frac{\sqrt{\tau} v_{2}}{\left(1+\sqrt{t^{\prime}} v_{1}\right) \ln \left(1+\sqrt{t^{\prime}} v_{1}\right)^{1 / b-1}}
$$

and thus

$$
z=\frac{1}{b} \frac{\sqrt{\tau}}{\sqrt{t^{\prime}} \ln \sqrt{t^{\prime}}} v
$$

where $v$ is again a variable with a Cauchy distribution (we have not determined the exact form using the wall). This is compatible with a Lorentzian aging packet but with:

$$
\Gamma=\frac{1}{b} \frac{\sqrt{\tau}}{\sqrt{t^{\prime}} \ln \sqrt{t^{\prime}}}
$$

consistent with:

$$
\Gamma=f\left[\frac{h(t)}{h\left(t^{\prime}\right)}\right] \quad h(t)=\exp \left(C(\ln t)^{1 / b}\right)
$$

with $a<1$, again performing an expansion in small $\tau / t_{w}$ and with $\beta=1 / 2$.

\section{CONCLUSION}

In this paper we have investigated two time quantities in several one dimensional diffusion models with random and non random environments. These quantities are functions of the waiting time $t^{\prime}=t_{w}$ after the initial localized condition at $t=0$ and a later time $t$. The (averaged) distribution $Q\left(z, t, t^{\prime}\right)$ of relative displacements $z=x(t)-x\left(t^{\prime}\right)$ between $t$ and $t^{\prime}$ was studied. Part of this study was numerical (in Sinai's model) and we have reached times up to $10^{15}$. Our results showed that the times reached in a previous simulation ${ }^{34}$ were vastly insufficient. Our conclusions are different from the one of ${ }^{34}$. Part of the study was analytical: we have computed two time quantities for a directed model related to the biased Sinai model and we have introduced several new models which can be studied analytically. Our main results are the following.

We have identified three generic regimes for large times $t_{w} \rightarrow \infty, t \rightarrow \infty$ :

(i) At small separations $\tau=t-t_{w} \ll t_{w}$ a quasi-equilibrium regime. Evidence for that regime was found in Sinai's model. In that regime $\overline{Q\left(z, t_{w}+\tau, t_{w}\right)}$ reaches a limit $\overline{Q(z, \tau)}$ for $t_{w} \rightarrow \infty$. We have argued, and checked numerically, that this distribution has some peculiarities. For large $\tau$ it does admit a limit $\overline{Q(z)}$ but this limit exhibits an algebraic tail originating from rare configurations of the disorder. The moments of the relative displacement $\overline{\left\langle z^{n}(\tau)\right\rangle}$ with 
$n>1 / 2$ grow unboundedly with $\tau$. We have also proposed an expression for the distribution $\overline{Q(z, \tau)}$ based on arguments on periodic media.

We have also concluded, from our simulations and from physical arguments, that in Sinai's model usual equilibrium theorems hold in this quasi equilibrium regime (TTI and FDT). We have obtained a generalized expression of these theorems to probability distributions such as $Q\left(z, t, t^{\prime}\right)$, and shown that in this regime $Q(z, \tau)$ and the response function $R(z, \tau)$ obey and exact differential relation. That these theorems should hold in that regime is confirmed by recently obtained rigorous bounds, as we have discussed. This unveils an interesting situation of a quasi equilibrium regime with a lot of internal structure, wide fluctuations, and internal logarithmic diffusion (moments growing with $\tau)$ which calls for further studies.

(ii) at large time separations, $L(t) \sim L\left(t_{w}\right)$ there is a diffusion regime. There the displacements scale as $x(t) \sim$ $x\left(t_{w}\right) \sim L(t)$ and there are scaling forms for the probability distributions. In the model of Section $(\mathrm{V})$ we have obtained this regime analytically.

(ii) finally there is an intermediate aging regime. One should first look at this regime in the probability of staying in a finite neighborhood $z$ of the same point between $t$ and $t^{\prime}$ which is generically of the form

$$
Q\left(z, t, t^{\prime}\right)=F\left[z, \frac{h(t)}{h\left(t^{\prime}\right)}\right]
$$

In Sinai's model with a bias (and in the directed model with algebraic distribution of waiting times) one has aging with $h(t)=t$. If the waiting times are even more widely distributed, we find (159) (for $z=0$ ) with a large class of functions $h(t) \gg t$. Similarly we also find this behaviour in a solvable model in (V) where a large class of functions $h(t)$ (including subaging $h(t) \ll t$ ) can be obtained.

In the symmetric Sinai model (without a bias) we have found strong numerical evidence for the aging behaviour (159) with $h(t) \sim \ln t$ (for small finite $z$ ). In that model we have even found a more striking result:

$$
Q\left(z, t, t^{\prime}\right)=Q_{0}(z) f\left[\frac{h(t)}{h\left(t^{\prime}\right)}\right]
$$

i.e a decoupled form for the aging regime. This suggests an interpretation of the aging regime in Sinai's model as well equilibrated well which get emptied on aging time scales. This picture should be checked further.

Another consequence of our result for Sinai's model is that, since the aging regime must be compatible with the diffusion one and we have found that $h(t) \sim L(t) \sim \ln t$, there must be a singularity in the two time diffusion front at $z=0$ to allow for a non trivial aging regime.

We have given a general explanation of these regimes using scaling arguments on the next highest barrier encountered by the particle. This allows to understand the aging form in Sinai's model. It also strongly suggests that the aging in Sinai's model could be studied analytically by only computing the distribution of next highest barrier (a purely geometrical feature of the energy landscape). These considerations also lead to define a class of models for which the distribution of these barriers can be computed exactly, and allows for predictions of the aging forms (159).

Though we did get a consistent picture of aging in Sinai's model, we cannot rule out completely other regimes. For instance, we have not explored in more details the behaviour of the moments of the displacement in Sinai's model. As in Section III one could say that at the very beginning of the aging regime (i.e $\ln t / \ln t_{w} \sim 1+\epsilon$ fixed, a small fraction of particles have escaped from their well and have experienced Sinai's diffusion to another well. One then gets:

$$
\overline{\left|<x(t)-x\left(t_{w}\right)>\right|^{n}} \sim\left(\frac{\ln t}{\ln t_{w}}-1\right)^{\beta}\left(\ln \left(t-t_{w}\right)\right)^{2 n} \sim\left(\ln t_{w}\right)^{2 n-\beta}\left(\ln t-\ln t_{w}\right)^{\beta}
$$

Thus by the same mechanism as in Section III the various moments may have some different aging behaviours. We have not attempted to obtain a precise estimate for $\beta$ but a rough estimate from our numerical simulations (Fig. 13 ) is consistent with $\beta=1$. If this is the case the moment $n=1 / 2$ may have an aging behaviour as $t / t_{w}$. The general issue of the matching bewteen the three regimes defined here deserves to be investigated further.

Another open problem is the behaviour of the response in these regimes. It is important to determine how to define properly, and study beyond mean field the way the equilibrium theorems are violated. Since, as we have shown, sample to sample fluctuations play a strong role and one should focus on distributions, one needs extensions of the mean field ideas. As a first step we have given analytical expressions and definitions of quantities adapted to low dimension and which measure these violations. A detailed numerical and further analytical investigation of these quantities is deferred to the future.

To summarize, we have found that some of the concepts defined in mean field are still useful in low dimensional models, though they have to be seriously adapted. We hope that this study will also help understand dynamical 
behaviour in low dimensional but more complex systems such as domain wall motion with disorder and coarsening in random spin systems, where ultra slow anomalous diffusion processes are expected to play a crucial role. We expect that the various regimes defined here should be present in these systems as well.

We thank A. Barrat, A. Georges, L. Cugliandolo and J. Kurchan for useful discussions.

\section{APPENDIX A: DISCRETE VERSION OF SINAI MODEL AND DIAGONALIZATION}

In this Appendix we describe in detail the observables for discrete hopping models. We also describe the numerical method used in the paper. We establish some FDT relations and other useful exact relations for discrete models.

Let us consider the Fokker Planck operator $H_{F P}$ defined in (7). It can be written as:

$$
\left(H_{F P}\right)_{n, m} P_{m}=\frac{d P_{n}}{d t}=-\left(J_{n+1, n}-J_{n, n-1}\right)
$$

where the current flowing from site $n-1$ to $n$ is by definition:

$$
J_{n, n-1}=e^{\phi_{n}} P_{n-1}-e^{-\phi_{n}} P_{n}
$$

We need to compute the Green function $P\left(n, t \mid n_{0}, t_{0}\right)\left(t \geq t_{0}\right)$ which is defined as the solution of (7) with initial condition $P\left(n, t_{0} \mid n_{0}, t_{0}\right)=\delta_{n n_{0}}$. Following Ref. ${ }^{29}$ it is useful to map the FP equation onto a Schrödinger equation corresponding to a symmetric matrix. One has:

$$
P\left(n, t \mid n_{0}, t_{0}\right)=e^{-\frac{1}{2}\left(U_{n}-U_{n_{0}}\right)} \sum_{\alpha} \psi_{n}^{\alpha} \psi_{n_{0}}^{\alpha} e^{-E_{\alpha} t}
$$

where the $\psi^{\alpha}$ are the eigenstates of the Schrödinger operator:

$$
\left(H_{s}\right)_{n, m} \psi_{m}^{\alpha}=-\left(\psi_{n+1}^{\alpha}+\psi_{n-1}^{\alpha}-2 \psi_{n}^{\alpha}\right)+V_{n} \psi_{n}^{\alpha}=E_{\alpha} \psi_{n}^{\alpha}
$$

in the potential:

$$
V_{n}=e^{\phi_{n+1}}+e^{-\phi_{n}}-2
$$

As discussed in Ref. ${ }^{29}$ the random operator $H_{s}$, which is a version of supersymmetric quantum mechanics, is quite peculiar : all states are localized but the spectrum of $H_{s}$ is positive, without the Lifschitz tails usually associated to random one-dimensional potentials. An eigenfunction corresponding to the energy level $E_{0}=0$ is always exactly known, i.e $\psi_{n}^{0}=Z e^{-U_{n} / 2}$. Whether or not this is the actual ground state depends on whether $\psi_{n}^{0}$ is normalizable, i.e on the boundary conditions. This is also related to the breaking of supersymmetry.

The Schrödinger operator is a tridiagonal symmetric matrix and is easily diagonalized for large size $L$. Let us first consider the problem with $L+1$ sites $k=0, L$ with reflexive boundaries. The same change of function $P_{n}=e^{U_{n} / 2} \psi_{n}$ is used and formula (A3) holds.

$$
\begin{aligned}
\left(H_{F P}\right)_{i j}= & \delta_{i+1, j} e^{-\phi_{j}}+\delta_{i-1, j} e^{\phi_{j+1}}-\delta_{i, j}\left(e^{-\phi_{i}}+e^{\phi_{i+1}}\right) \\
& \left(H_{F P}\right)_{0 j}=\delta_{1, j} e^{-\phi_{1}}-\delta_{0, j} e^{\phi_{1}} \\
\left(H_{s}\right)_{i j}=-\left(\delta_{i+1, j}+\delta_{i-1, j}\right)+\delta_{i, j}\left(e^{-\phi_{i}}+e^{\phi_{i+1}}\right) & \left(H_{s}\right)_{0 j}=-\delta_{1, j}+\delta_{0, j} e^{\phi_{1}}
\end{aligned}
$$

these boundary conditions simply amount to choose $\phi_{-1}=+\infty$ and $\phi_{L+1}=-\infty$ and restrict the problem to sites $k=0, . . L$.

explicit expressions of quantities of interest and FDT theorems in discrete version

The two time quantities of interest are correlation functions of some operator $O\left(n, n^{\prime}\right)$ :

$$
\begin{aligned}
O\left(t, t^{\prime}\right)= & <O\left(x(t), x\left(t^{\prime}\right)\right)>=\sum_{n, n^{\prime}} O\left(n, n^{\prime}\right) P\left(n, t \mid n^{\prime}, t^{\prime}\right) P\left(n^{\prime}, t^{\prime} \mid n_{0}, t_{0}=0\right) \\
& =\sum_{\alpha, \beta} e^{-E_{\alpha}\left(t-t^{\prime}\right)-E_{\beta} t^{\prime}} \sum_{n} \sum_{n^{\prime}} O\left(n, n^{\prime}\right) e^{-U_{n} / 2} \psi_{n}^{\alpha} \psi_{n^{\prime}}^{\alpha} \psi_{n^{\prime}}^{\beta} e^{U_{n_{0}} / 2} \psi_{n_{0}}^{\beta}
\end{aligned}
$$


and response functions of some operator $R\left(n, n^{\prime}\right)$

$$
R\left(t, t^{\prime}\right)=\frac{\delta<R\left(x(t), x\left(t^{\prime}\right)\right)>_{f}}{\delta f\left(t^{\prime}\right)}
$$

defined by adding a short-duration pulse of small uniform force of integrated strength $f$, i.e $\phi_{n} \rightarrow \phi_{n}+f / 2$ at time $t^{\prime}$. Using the definition $P\left(n, t \mid n^{\prime}, t^{\prime}\right)=\left(e^{H_{F P}\left(t-t^{\prime}\right)}\right)_{n n^{\prime}}$ one has:

$$
R^{+}\left(t, t^{\prime}\right)=\frac{1}{2} \sum_{n, n^{\prime}, m^{\prime}} R\left(n, n^{\prime}\right) P\left(n, t \mid n^{\prime}, t^{\prime}\right) B_{n^{\prime} m^{\prime}} P\left(m^{\prime}, t^{\prime} \mid n_{0}, t_{0}=0\right)
$$

if the pulse is at $t^{\prime}-\epsilon$, or

$$
R\left(t, t^{\prime}\right)=\frac{1}{2} \sum_{n, n^{\prime}, m} R\left(n, n^{\prime}\right) P\left(n, t \mid m, t^{\prime}\right) B_{m n^{\prime}} P\left(n^{\prime}, t^{\prime} \mid n_{0}, t_{0}=0\right)
$$

if the pulse is at $t^{\prime}+\epsilon$ (Ito).

We have defined

$$
B_{m m^{\prime}}=\sum_{n^{\prime}} \frac{\left(\delta H_{F P}\right)_{m m^{\prime}}}{\delta \phi_{n^{\prime}}}=\delta_{m, m^{\prime}}\left(e^{-\phi_{m}}-e^{\phi_{m+1}}\right)+\delta_{m-1, m^{\prime}} e^{\phi_{m}}-\delta_{m+1, m^{\prime}} e^{-\phi_{m+1}}
$$

We will now establish (i) the FDT relations valid when equilibrium is attained (ii) some exact relations always valid. These equations will relate the correlation functions of the operator $O\left(n, n^{\prime}\right)$ with response function of the operator $R\left(n, n^{\prime}\right)$. There must be a relation between $R$ and $O$ for these to hold, which is:

$$
\frac{1}{2}(R(n, m)+R(n, m-1))=O(n, m)-O(n, m-1)
$$

This relation generalizes the usual relation $R\left(x, x^{\prime}\right)=\partial_{x^{\prime}} O\left(x, x^{\prime}\right)$ (see next Section) valid for continuous systems. We start with the following identity:

$$
\frac{\partial O\left(t, t^{\prime}\right)}{\partial t^{\prime}}=\sum_{n, n^{\prime}, m, m^{\prime}} O\left(n, n^{\prime}\right) P\left(n, t \mid m, t^{\prime}\right) H_{m m^{\prime}}\left(\delta_{m n^{\prime}}-\delta_{m^{\prime} n^{\prime}}\right) P\left(m^{\prime}, t^{\prime} \mid n_{0}, t_{0}=0\right)
$$

Note the simplification:

$$
H_{m m^{\prime}}\left(\delta_{m n^{\prime}}-\delta_{m^{\prime} n^{\prime}}\right)=\left(\delta_{m+1, m^{\prime}} e^{-\phi_{m^{\prime}}}+\delta_{m-1, m^{\prime}} e^{\phi_{m^{\prime}+1}}\right)\left(\delta_{m n^{\prime}}-\delta_{m^{\prime} n^{\prime}}\right)
$$

Now the following exact relation can be established:

$$
\sum_{n^{\prime}, m^{\prime}} O\left(n, n^{\prime}\right) H_{m m^{\prime}}\left(\delta_{m n^{\prime}}-\delta_{m^{\prime} n^{\prime}}\right) P_{m^{\prime}}=\frac{1}{2} \sum_{n^{\prime}} R\left(n, n^{\prime}\right) B_{m n^{\prime}} P_{n^{\prime}}+\frac{1}{2} R(n, m)\left(J_{m+1, m}+J_{m, m-1}\right)
$$

for any set of $P_{m}$, provided the above relation (A12) holds between the operators $O$ and $R$ ( $J$ being defined as in (A2)). It yields to:

$$
\frac{\partial O\left(t, t^{\prime}\right)}{\partial t^{\prime}}=R\left(t, t^{\prime}\right)+\frac{1}{2} \sum_{n, m} R(n, m) P\left(n, t \mid m, t^{\prime}\right)\left(J_{m+1, m}\left(t^{\prime}\right)+J_{m, m-1}\left(t^{\prime}\right)\right)
$$

where $J_{m, m-1}\left(t^{\prime}\right)=e^{\phi_{m}} P\left(m-1, t^{\prime} \mid n_{0}, t_{0}=0\right)-e^{-\phi_{m}} P\left(m, t^{\prime} \mid n_{0}, t_{0}=0\right)$ and we are using the Ito response.

When equilibrium is attained, i.e either in the limit $t^{\prime} \rightarrow+\infty$ before $L \rightarrow+\infty$ or, if there is an FDT regime in the problem (see text, this usually entails averaging all these correlations over disorder), then one can set the current to zero, $J=0$. Then the the fluctuation-dissipation relation holds:

$$
\frac{\partial O\left(t, t^{\prime}\right)}{\partial t^{\prime}}=R\left(t, t^{\prime}\right)
$$

applications 
- The choice $O(m, n)=n m$ and $R(n, m)=n$ is consistent with (A12). It gives:

$$
\frac{\partial<x(t) x\left(t^{\prime}\right)>}{\partial t^{\prime}}=\frac{\delta<x(t)>_{f}}{\delta f\left(t^{\prime}\right)}
$$

- The choice $O(m, n)=(n-m)^{2}$ and $R(n, m)=-2(n-m)$ is consistent with (A12). It gives:

$$
\frac{\partial<\left(x(t)-x\left(t^{\prime}\right)\right)^{2}>}{\partial t^{\prime}}=-2 \frac{\delta<\left(x(t)-x\left(t^{\prime}\right)>_{f}\right.}{\delta f\left(t^{\prime}\right)}
$$

- The general choice:

$$
O(n, m)=\frac{1}{2}(\tilde{O}(n, m)+\tilde{O}(n, m+1)) \quad R(n, m)=\tilde{O}(n, m+1)-\tilde{O}(n, m)
$$

satisfies the condition (A12).

Thus one can choose $\tilde{O}(n, m)=\delta_{z-(n-m)}$ and obtain the exact relation:

$$
\begin{aligned}
& \partial_{t^{\prime}} \frac{1}{2}\left(Q\left(z, t, t^{\prime}\right)+Q\left(z+1, t, t^{\prime}\right)\right)=R\left(z+1, t, t^{\prime}\right)-R\left(z, t, t^{\prime}\right) \\
& +\frac{1}{2} \sum_{m}\left(P\left(z+1+m, t \mid m t^{\prime}\right)-P\left(z+m, t \mid m t^{\prime}\right)\right)\left(J_{m+1, m}\left(t^{\prime}\right)+J_{m, m-1}\left(t^{\prime}\right)\right)
\end{aligned}
$$

This is the discrete equivalent of the continuous relation derived in the next Section (B23).

Finally, one can wonder what happens when a bias is applied on a finite size periodic ring. There a stationary distribution with a fixed current is reached at large $t^{\prime}$. There is an extension of the FDT theorem. Indeed one has:

$$
\frac{\partial O\left(t, t^{\prime}\right)}{\partial t^{\prime}}=R\left(t, t^{\prime}\right)+J \sum_{n, m} R(n, m) P\left(n, t \mid m, t^{\prime}\right)
$$

Note that $J$ is simply related to the velocity (see e.g ${ }^{46}$ ).

\section{APPENDIX B: FDT AND USEFUL EXACT RELATIONS FOR PROBABILITY DISTRIBUTIONS}

In this Appendix we derive a generalization of the FDT theorem on the probability distribution. It can then be used as a generating functional to obtain a hierarchy of FDT relations on all moments of the type $<x(t)^{n} x\left(t^{\prime}\right)^{m}>$.

We are interested in the joint probability that the particle is in $x$ at $t^{\prime}$ and then in $x$ at $t$.

$$
\hat{P}\left(x t, x^{\prime} t^{\prime} \mid x_{0} t_{0}\right)=P\left(x, t \mid x^{\prime}, t^{\prime}\right) P\left(x^{\prime}, t^{\prime} \mid x_{0}, 0\right)
$$

Let us recall the forward and backward FP equations:

$$
\begin{aligned}
& \partial_{t} P\left(x t \mid x^{\prime} t^{\prime}\right)=T \partial_{x} D(x) \partial_{x} P\left(x t \mid x^{\prime} t^{\prime}\right)-\partial_{x} D(x) F(x) P\left(x t \mid x^{\prime} t^{\prime}\right) \\
& \partial_{t^{\prime}} P\left(x t \mid x^{\prime} t^{\prime}\right)=-T \partial_{x^{\prime}} D\left(x^{\prime}\right) \partial_{x^{\prime}} P\left(x t \mid x^{\prime} t^{\prime}\right)-D\left(x^{\prime}\right) F\left(x^{\prime}\right) \partial_{x^{\prime}} P\left(x t \mid x^{\prime} t^{\prime}\right)
\end{aligned}
$$

We now derive the differential equation for the joint probability $\hat{P}$ :

$$
\begin{gathered}
\partial_{t^{\prime}} \hat{P}=\left(\partial_{t^{\prime}} P\left(x, t \mid x^{\prime}, t^{\prime}\right)\right) P\left(x^{\prime}, t^{\prime} \mid 0,0\right)+P\left(x, t \mid x^{\prime}, t^{\prime}\right)\left(\partial_{t^{\prime}} P\left(x^{\prime}, t^{\prime} \mid 0,0\right)\right) \\
\partial_{t^{\prime}} \hat{P}=-\int d y d y^{\prime} P\left(x, t \mid y, t^{\prime}\right)\left(\left[H_{F P}, \delta_{x^{\prime}}\right]\right)_{y y^{\prime}} P\left(y^{\prime}, t^{\prime} \mid 0,0\right)
\end{gathered}
$$

with:

$$
\left[H_{F P}, \delta_{x^{\prime}}\right]=\left[T \partial D \partial-\partial D F, \delta_{x^{\prime}}\right]=T \partial D\left[\partial, \delta_{x^{\prime}}\right]+\left[\partial, \delta_{x^{\prime}}\right](T D \partial-D F)
$$

The last term is the current $J=-(T D \partial-D F) P$. In the FDT regime (for large $t^{\prime}$ ) the current is expected to vanish and we are left with the following equation for $\hat{P}$ : 


$$
\begin{array}{r}
\partial_{t^{\prime}} \hat{P}=-\int d y d y^{\prime} P\left(x, t \mid y, t^{\prime}\right)\left(T \partial D\left[\partial, \delta_{x^{\prime}}\right]\right)_{y y^{\prime}} P\left(y^{\prime}, t^{\prime} \mid 0,0\right) \\
\partial_{t^{\prime}} \hat{P}=\partial_{x^{\prime}} \int d y d y^{\prime} P\left(x, t \mid y, t^{\prime}\right)\left(T \partial D \delta_{x^{\prime}}\right)_{y y^{\prime}} P\left(y^{\prime}, t^{\prime} \mid 0,0\right) \\
\partial_{t^{\prime}} \hat{P}=-T \partial_{x^{\prime}}\left[\left(\partial_{x^{\prime}} P\left(x, t \mid x^{\prime}, t^{\prime}\right)\right) D\left(x^{\prime}\right) P\left(x^{\prime}, t^{\prime} \mid 0,0\right)\right]
\end{array}
$$

finally we obtain:

$$
\partial_{t^{\prime}} \hat{P}=-T \partial_{x^{\prime}}^{2}\left(D\left(x^{\prime}\right) \hat{P}\right)+T \partial_{x^{\prime}}\left(P\left(x, t \mid x^{\prime}, t^{\prime}\right) \partial_{x^{\prime}}\left(D\left(x^{\prime}\right) P\left(x^{\prime}, t^{\prime} \mid 0,0\right)\right)\right)
$$

and since $\frac{\delta H_{F P}}{\delta h\left(t^{\prime}\right)}=-\partial_{x^{\prime}} D\left(x^{\prime}\right)$

$$
\partial_{t^{\prime}} \hat{P}=-T \partial_{x^{\prime}}^{2}\left(D\left(x^{\prime}\right) \hat{P}\right)-T \partial_{x^{\prime}} \frac{\delta \hat{P}_{h}^{+}}{\delta h\left(t^{\prime}\right)}
$$

where we define $\hat{P}_{h}^{+}\left(x t\left|x^{\prime} t^{\prime}\right| x_{0} 0\right)$ the joint probability when a field pulse has been applied at $t^{\prime}-\epsilon$. This is the equation which relates exactly the joint probability distribution $\hat{P}\left(x t, x^{\prime} t^{\prime} \mid x_{0} t_{0}\right)$ to the response distribution in the quasi-equilibrium FDT regime. It was obtained by setting the current at time $t^{\prime}$ to zero.

A similar equation can be derived for the field applied at time $t^{\prime}+\epsilon$. From (B10) one has also that:

$$
\partial_{t^{\prime}} \hat{P}=-T \partial_{x^{\prime}} \frac{\delta \hat{P}_{h}}{\delta h\left(t^{\prime}\right)}
$$

where $\hat{P}_{h}\left(x t\left|x^{\prime} t^{\prime}\right| x_{0} 0\right)$ the joint probability when a field pulse has been applied at $t^{\prime}+\epsilon$. This corresponds to Ito's prescription for the response functions since $\delta \hat{P}_{h}\left(x t^{\prime}\left|x^{\prime} t^{\prime}\right| x_{0} 0\right) / \delta h\left(t^{\prime}\right)=0$.

From this it is immediate to derive a similar FDT equation for the probability function $Q\left(z, t, t^{\prime}\right)=\int d x d x^{\prime} \delta(z-$ $\left.x+x^{\prime}\right) \hat{P}\left(x t, x^{\prime} t^{\prime} \mid x_{0} t_{0}\right)$. Multiplying the above equation by the delta function, integrating with respect to $\mathrm{x}$ and $\mathrm{x}$, and integrating by parts one gets:

$$
\partial_{t^{\prime}} Q\left(z, t, t^{\prime}\right)=-T \partial_{z}^{2} Q\left(z, t, t^{\prime}\right)+T \partial_{z} \frac{\delta Q_{h}^{+}\left(z, t, t^{\prime}\right)}{\delta h\left(t^{\prime}\right)}+T \partial_{z} \int d x_{1} d x_{2} \delta\left(z-x_{1}+x_{2}\right) P\left(x_{1}, t \mid x_{2}, t^{\prime}\right) J\left(x_{2}\right)
$$

in the the long time regime $t^{\prime} \rightarrow \infty$ the current vanishes and we are left with the FDT regime of the equation:

$$
\partial_{t^{\prime}} Q\left(z, t, t^{\prime}\right)=-T \partial_{z}^{2} Q\left(z, t, t^{\prime}\right)+T \partial_{z} \frac{\delta Q_{h}^{+}\left(z, t, t^{\prime}\right)}{\delta h\left(t^{\prime}\right)}
$$

Similarly with the Ito prescription:

$$
\partial_{t^{\prime}} Q\left(z, t, t^{\prime}\right)=T \partial_{z} \frac{\delta Q_{h}\left(z, t, t^{\prime}\right)}{\delta h\left(t^{\prime}\right)}
$$

We can check that this equation is more general than the conventional FDT theorem, and indeed gives back the usual FDT result. Defining:

$$
B\left(t, t^{\prime}\right)_{F D T}=\int d z z^{2} Q_{F D T}\left(z, t, t^{\prime}\right)
$$

and inserting it in the generalized equation gives:

$$
\partial_{t^{\prime}} B\left(t, t^{\prime}\right)=-T \int d z z^{2} \partial_{z}^{2} Q\left(z, t, t^{\prime}\right)+T \int d z z^{2} \partial_{z} \frac{\delta Q_{h}^{+}\left(z, t, t^{\prime}\right)}{\delta h\left(t^{\prime}\right)}
$$

after integration by part this gives simply:

$$
\partial_{t^{\prime}} B\left(t, t^{\prime}\right)=-2 T-2 T \frac{\delta}{\delta h\left(t^{\prime}\right)} \int d z z Q_{h}^{+}\left(z, t, t^{\prime}\right)=-2 T\left(1+R^{+}\left(t, t^{\prime}\right)-R^{+}\left(t^{\prime}, t^{\prime}-\epsilon\right)\right)=-2 T R\left(t, t^{\prime}\right)
$$

With the Ito prescription one has simply: 


$$
\partial_{t^{\prime}} B\left(t, t^{\prime}\right)=-2 T \frac{\delta}{\delta h\left(t^{\prime}\right)} \int d z z Q_{h}\left(z, t, t^{\prime}\right)=-2 T R\left(t, t^{\prime}\right)
$$

using $R\left(t^{\prime}, t^{\prime}\right)=R\left(t^{\prime}, t^{\prime}+\epsilon\right)=0$.

A motivation is to find a generalized form to this equation which would be valid in the aging regime as well.

\section{exact relations and FDT violation ratios}

It is useful also to give the exact relations (always valid) for averages of operators. They allow to obtain explicit the FDT violation ratios.

We put back the current term that we have neglected. We obtain then instead of (B10) the (still) exact relation:

$$
\partial_{t^{\prime}} \hat{P}+T \partial_{x^{\prime}}^{2}\left(D\left(x^{\prime}\right) \hat{P}\right)-T \partial_{x^{\prime}}\left(P\left(x, t \mid y, t^{\prime}\right) \partial_{x^{\prime}} D\left(x^{\prime}\right) P\left(x^{\prime}, t^{\prime} \mid 0,0\right)\right)=\int d y d y^{\prime} P\left(x, t \mid y, t^{\prime}\right)\left[\partial, \delta_{x^{\prime}}\right]_{y y^{\prime}} J\left(y^{\prime} t^{\prime} \mid 00\right)
$$

with $J\left(y^{\prime} t^{\prime} \mid 00\right)=-D\left(y^{\prime}\right)\left(T \partial_{y^{\prime}}-F\left(y^{\prime}\right)\right) P\left(y^{\prime} t^{\prime} \mid 00\right)$. We will use the Ito response here.

Let us study a general observable $O\left(x, x^{\prime}\right)$. Multiplying the above equation, integrating over $x$ and $x^{\prime}$, and integrating by parts (assuming no contributions from boundaries) one has an exact relation which relates the averages of of $O\left(x, x^{\prime}\right)$ and of $O^{\prime}\left(x, x^{\prime}\right)=\partial_{x^{\prime}} O\left(x, x^{\prime}\right)$. Defining:

$$
V_{0}\left(t, t^{\prime}\right)=\partial_{t^{\prime}}<O\left(x(t), x\left(t^{\prime}\right)\right)>-T \frac{\delta<O^{\prime}\left(x, x^{\prime}\right)>_{h}}{\delta h\left(t^{\prime}\right)} \equiv\left(1-X_{O}\left(t, t^{\prime}\right)\right) \partial_{t^{\prime}}<O>\left(t, t^{\prime}\right)
$$

the relation reads:

$$
V_{0}\left(t, t^{\prime}\right)=\int d x d x^{\prime} P\left(x, t \mid x^{\prime}, t^{\prime}\right) O^{\prime}\left(x, x^{\prime}\right) J\left(x^{\prime} t^{\prime} \mid 00\right)
$$

We have defined above the FDT violation ratio $X_{0}$ associated with the operator $O$. If the other response was used there would be in addition a term $-T<D\left(x\left(t^{\prime}\right)\right) O_{2}\left(x(t), x\left(t^{\prime}\right)\right)>$ in the above equation with $O_{2}\left(x, x^{\prime}\right)=\partial_{x^{\prime}}^{2} O\left(x, x^{\prime}\right)$. Again for the Ito response the second derivative term is absent.

We will also give an exact relation for the relative displacements. Let us consider an operator $O(z)$. Then one has:

$$
\partial_{t^{\prime}}<O(z)>+T \frac{\delta<O^{\prime}(z)>_{h}}{\delta h\left(t^{\prime}\right)}=\int d x_{1} d x_{2} O^{\prime}\left(x_{1}-x_{2}\right) P\left(x_{1} t \mid x_{2} t^{\prime}\right) J\left(x_{2} t^{\prime} \mid 00\right)
$$

And thus one can define also a generalized FDT ratio:

$$
\tilde{X}_{O}\left(t, t^{\prime}\right)=1-\frac{\int d x_{1} d x_{2} O^{\prime}\left(x_{1}-x_{2}\right) P\left(x_{1} t \mid x_{2} t^{\prime}\right) J\left(x_{2} t^{\prime} \mid 00\right)}{\partial_{t^{\prime}}<O(z)>}
$$

\section{APPENDIX C: BOUNDS}

Here we illustrate the bounds recently proposed by $\mathrm{CDK}^{40}$. We use the framework of the generalized FDT relation of the preceding section and our derivation is thus technically slightly different, though identical in spirit to ${ }^{40}$. We work directly with the FP equation and a space dependent diffusion coefficient.

The nice observation of CDK is that the current which appear in (B22) also appears in the $H$ theorem which states that the free energy:

$$
H\left(t^{\prime}\right)=\int d x^{\prime} P\left(x^{\prime} t^{\prime} \mid 00\right)\left(T \ln P\left(x^{\prime}, t^{\prime} \mid 00\right)-U\left(x^{\prime}\right)\right)
$$

is always decreasing with:

$$
\frac{d H\left(t^{\prime}\right)}{d t^{\prime}}=-\int d x^{\prime} \frac{\left(J\left(x^{\prime} t^{\prime} \mid 00\right)\right)^{2}}{D\left(x^{\prime}\right) P\left(x^{\prime}, t^{\prime} \mid 00\right)}
$$

The CKS bounding amounts to bound $V_{0}\left(t, t^{\prime}\right)$ defined in (B21) by: 


$$
\begin{aligned}
& \left|V_{0}\left(t, t^{\prime}\right)\right| \leq\left|\frac{d H\left(t^{\prime}\right)}{d t^{\prime}}\right|^{1 / 2} \int d x d x^{\prime} P\left(x, t \mid x^{\prime}, t^{\prime}\right) O^{\prime}\left(x, x^{\prime}\right)^{2} D\left(x^{\prime}\right) P\left(x^{\prime}, t^{\prime} \mid 00\right) \\
& =\left|\frac{d H\left(t^{\prime}\right)}{d t^{\prime}}\right|^{1 / 2}<\left(O^{\prime}\left(x(t), x\left(t^{\prime}\right)\right)^{2} D\left(x\left(t^{\prime}\right)\right)>^{1 / 2}\right.
\end{aligned}
$$

using the Cauchy-Schwartz CS inequality and $\int d x P\left(x, t \mid x^{\prime} t^{\prime}\right)=1$

Note also that disorder averages can be bounded similarly by applying the CS inequality at the same time to the integrals over $x, x^{\prime}$ and configurations.

One then gets:

$$
\left|\overline{V_{0}\left(t, t^{\prime}\right)}\right| \leq\left|\frac{d \bar{H}\left(t^{\prime}\right)}{d t^{\prime}}\right|^{1 / 2}\left|\overline{<O^{\prime}\left(x(t), x\left(t^{\prime}\right)\right)^{2} D\left(x\left(t^{\prime}\right)\right)>}\right|^{1 / 2}
$$

Or, as pointd out by CDK, in an integrated version:

$$
\left|\int_{t^{\prime}}^{t} d s \overline{V_{0}(t, s)}\right| \leq \int_{t^{\prime}}^{t} d s\left(\left|\frac{d \bar{H}(s)}{d s}\right|^{1 / 2}\left|\overline{\left\langle O^{\prime}(x(t), x(s))^{2} D(x(s))>\right.}\right|^{1 / 2}\right)
$$

If we choose $O\left(x, x^{\prime}\right)=x x^{\prime}$ and $D(x)=1$ one gets:

$$
\left|\overline{\left\langle x^{2}(t)>\right.}-\overline{<x(t) x\left(t^{\prime}\right)>}-T \int_{t^{\prime}}^{t} R\left(t, t^{\prime}\right)\right| \leq\left(\overline{\left\langle x^{2}(t)>\right.}\right)^{1 / 2} \int_{t^{\prime}}^{t} d s\left(\left|\frac{d \bar{H}(s)}{d s}\right|^{1 / 2}\right.
$$

One can also derive bounds using (B23) for the relative displacements. Using CS, (B23) leads to the bound:

$$
\left|\partial_{t^{\prime}}<O(z)>+T \frac{<\delta O^{\prime}(z)>_{h}}{\delta h\left(t^{\prime}\right)}\right| \leq \mid<\left(O^{\prime}\left(x(t)-x\left(t^{\prime}\right)\right)^{2}>\left.\right|^{1 / 2}\left|\frac{d \bar{H}\left(t^{\prime}\right)}{d t^{\prime}}\right|^{1 / 2}\right.
$$

Which can be rewritten as:

$$
\left|\partial_{t^{\prime}} \int d z O(z) Q\left(z, t, t^{\prime}\right)+T \frac{\delta}{\delta h\left(t^{\prime}\right)} \int d z O^{\prime}(z) \frac{\delta Q_{h}\left(z, t, t^{\prime}\right)}{\delta h\left(t^{\prime}\right)}\right| \leq\left|\int d z O^{\prime 2}(z) Q\left(z, t, t^{\prime}\right)\right|^{1 / 2}\left|\frac{d \bar{H}\left(t^{\prime}\right)}{d t^{\prime}}\right|^{1 / 2}
$$

This yields in particular:

$$
\left|\partial_{t^{\prime}} B\left(t, t^{\prime}\right)+2 T R\left(t, t^{\prime}\right)\right| \leq B\left(t, t^{\prime}\right)^{1 / 2}\left|\frac{d \bar{H}\left(t^{\prime}\right)}{d t^{\prime}}\right|^{1 / 2}
$$

Or its integrated version:

$$
\left|-B\left(t, t^{\prime}\right)+2 T \int_{t^{\prime}}^{t} R\left(t, t^{\prime}\right)\right| \leq \int_{t^{\prime}}^{t} d s B(t, s)^{1 / 2}\left|\frac{d \bar{H}(s)}{d s}\right|^{1 / 2}
$$

More generally the bound can be used to constrain the $\tilde{X}_{O}$ defined in (B24):

$$
\left|1-\tilde{X}_{O}\left(t, t^{\prime}\right)\right| \leq \frac{<O^{\prime 2}>\left(t, t^{\prime}\right)\left|\frac{d \bar{H}\left(t^{\prime}\right)}{d t^{\prime}}\right|^{1 / 2}}{\left|\partial_{t^{\prime}}<O>\left(t, t^{\prime}\right)\right|}
$$

\section{APPENDIX D: MAPPINGS OF SEVERAL MODELS}

The method of change of variables allows to relate exactly members of a class of landscape. Let two landscapes and their corresponding Green's functions be:

$$
\begin{aligned}
\left(E_{b}(x), U(x)\right) & \leftrightarrow P\left(x, t \mid x_{0}, 0\right) \\
\left(E_{b}^{\prime}(x), U^{\prime}(x)\right) & \leftrightarrow P^{\prime}\left(x, t \mid x_{0}, 0\right)
\end{aligned}
$$

If there exist a function $y(x)$ such that: 


$$
E_{b}^{\prime}(x)=E_{b}(y(x))+2 \ln \frac{d y(x)}{d x} \quad U^{\prime}(x)=U(y(x))-\ln \frac{d y(x)}{d x}
$$

then the two Green functions are related through:

$$
P^{\prime}\left(x, t \mid x_{0}, 0\right)=\frac{d y(x)}{d x} P\left(y(x), t \mid y\left(x_{0}\right), 0\right)
$$

In particular one can map:

$$
(0, U(x)) \leftrightarrow(2 U(x(u)), 0)
$$

and

$$
\left(E_{b}(x), 0\right) \leftrightarrow\left(0, \frac{1}{2} E_{b}(x(u))\right)
$$

It is interesting in general because the new functions $U(x(u))$ or $E_{b}(x(u))$ are usually better behaved at large $u$.

Two general scenarios exist, confining potentials, unconfining ones. Let us take rapidly growing landscapes $\left(E_{b}(x)\right.$, $U(x))$ as in last section and map them onto $\left(0, U^{\prime}(u)\right)$. Then typically one has $U^{\prime}(u) \sim b \ln u$ at large $u$. The case $b 0$ is confining and corresponds to the case where barriers grow faster than valleys $E_{b}(x)-2 U(x)$. The case $b 0$ is fast diffusion and corresponds to the case where barriers grow slower than valleys $E_{b}(x)<-2 U(x)$.

Finally note that time dependent mappings could also be built using the following propagator:

$$
Q(u, t)=\frac{1}{\sqrt{4 \pi e^{t-t_{0}}-1}} e^{-\frac{\left(u-u_{0} e^{\left(t-t_{0}\right) / 2}\right)^{2}}{4\left(e^{t-t_{0}}-1\right)}}
$$

which satisfies:

$$
\partial_{t} Q(u, t)=\partial_{u} \partial_{u} Q(u, t)-\frac{1}{2} \partial_{u} u Q(u, t)
$$

\section{APPENDIX E: RESPONSE IN THE AGING MODEL}

We start from the exact equation obeyed by the Brownian diffusion propagator:

$$
R\left(u t \mid u^{\prime} t^{\prime}\right)=\frac{1}{\sqrt{4 \pi T\left(t-t^{\prime}\right)}} e^{-\frac{\left(u-u^{\prime}\right)^{2}}{4 T\left(t-t^{\prime}\right)}}
$$

The joint propagator

$$
\hat{R}\left(u t\left|u^{\prime} t^{\prime}\right| u_{0} t_{0}\right)=R\left(u t \mid u^{\prime} t^{\prime}\right) R\left(u^{\prime} t^{\prime} \mid u_{0} t_{0}\right)
$$

satisfies the exact equation:

$$
\begin{array}{r}
\partial_{t^{\prime}} \hat{R}=-T \partial_{u^{\prime}}^{2} \hat{R}+2 T \partial_{u^{\prime}}\left(R\left(u t \mid u^{\prime} t^{\prime}\right) \partial_{u^{\prime}} R\left(u^{\prime} t^{\prime} \mid u_{0} t_{0}\right)\right) \\
\partial_{t^{\prime}} \hat{R}=-2 T \partial_{u^{\prime}} \frac{\delta \hat{R}_{h}}{\delta h\left(t^{\prime}\right)}
\end{array}
$$

This is valid for the free (unbounded) brownian motion and yields for instance $R\left(t, t^{\prime}\right)=X \partial_{t^{\prime}} C\left(t, t^{\prime}\right)$ with $X=$ $1 /(2 T)$. A bounded brownian motion would instead converge to equilibrium with $X=1 / T$ and satisfy the FDT equation with $2 T$ replaced by $T$ in the last term.

One can now use the change of variable $\Phi(x) d / d x=d / d u$ and $\hat{R}\left(u t\left|u^{\prime} t^{\prime}\right| u_{0} t_{0}\right)=\Phi(x) \Phi\left(x^{\prime}\right) \hat{P}\left(x t\left|x^{\prime} t^{\prime}\right| x_{0} t_{0}\right)$ and obtain for the model studied previously, the exact equation valid in the unbounded case:

$$
\begin{aligned}
& \partial_{t^{\prime}} \hat{P}=-T \partial_{x^{\prime}}^{2}\left(\Phi\left(x^{\prime}\right)^{2} \hat{P}\right)+T \partial_{x^{\prime}}\left(P\left(x t \mid x^{\prime} t^{\prime}\right) \partial_{x^{\prime}}\left(\Phi\left(x^{\prime}\right)^{2} P\left(x^{\prime} t^{\prime} \mid x_{0} t_{0}\right)\right)\right) \\
& +T \partial_{x^{\prime}}\left(P\left(x t \mid x^{\prime} t^{\prime}\right) \Phi\left(x^{\prime}\right) \partial_{x^{\prime}}\left(\Phi\left(x^{\prime}\right) P\left(x^{\prime} t^{\prime} \mid x_{0} t_{0}\right)\right)\right)
\end{aligned}
$$

Since the diffusion coefficient is $D\left(x^{\prime}\right)=\Phi\left(x^{\prime}\right)^{2}$ in this model, and the response is $\frac{\delta H_{F P}}{\delta h\left(t^{\prime}\right)}=-\partial_{x^{\prime}} D\left(x^{\prime}\right)$ the above equation can be rewritten in a form very similar - but not identical - to the above general FDT equation: 


$$
\partial_{t^{\prime}} \hat{P}=-T \partial_{x^{\prime}} \frac{\delta \hat{P}_{h}}{\delta h\left(t^{\prime}\right)}-\partial_{x^{\prime}}\left(P\left(x t \mid x^{\prime} t^{\prime}\right) J\left(x^{\prime} t^{\prime}\right)\right)
$$

The last term cannot be simplified further and involves the current $J\left(x^{\prime} t^{\prime}\right)=-T \Phi\left(x^{\prime}\right) \partial_{x^{\prime}}\left(\Phi\left(x^{\prime}\right) P\left(x^{\prime} t^{\prime} \mid x_{0} t_{0}\right)\right)$ of the model. This equation is always valid for our model, even in the out of equilibrium regime.

The idea is that in the non trivial aging regime all three terms of the above equation will be roughly of the same order in $t^{\prime}$ and thus it will effectively lead to a non trivial FDT ratio $X\left(t, t^{\prime}\right)$.

One can use e.g this equation to study the correlation $C\left(t, t^{\prime}\right)=<x(t) x\left(t^{\prime}\right)>$. Multiplying by $x x^{\prime}$ and integrating over $x$ and $x^{\prime}$ one obtains:

$$
R\left(t, t^{\prime}\right) \equiv \frac{\delta<x(t)_{h}}{\delta h\left(t^{\prime}\right)}=X\left(t, t^{\prime}\right) \partial_{t^{\prime}}<x(t) x\left(t^{\prime}\right)>
$$

where we have defined:

$$
X\left(t, t^{\prime}\right)=\frac{1}{T}\left(1-\frac{\int d x d x^{\prime} x P\left(x t \mid x^{\prime} t^{\prime}\right) J\left(x^{\prime} t^{\prime}\right)}{\partial_{t^{\prime}}<x(t) x\left(t^{\prime}\right)>}\right)
$$

One can also define:

$$
X_{B}\left(t, t^{\prime}\right)=-\frac{1}{2} X_{C}\left(t, t^{\prime}\right)\left(1-\frac{\partial_{t^{\prime}}<x\left(t^{\prime}\right)^{2}}{2 \partial_{t^{\prime}}<x(t) x\left(t^{\prime}\right)}\right)^{-1}
$$

Introducing:

$$
\begin{aligned}
& A\left(t^{\prime}, \tau\right)=<x\left[\sqrt{t^{\prime}} u_{1}+\sqrt{\tau} u_{2}\right] u_{1} \Phi\left[x\left[\sqrt{t^{\prime}} u_{1}\right]\right] \\
& D\left(t^{\prime}, \tau\right)=<\left(u_{1}-u_{2} \sqrt{\frac{t^{\prime}}{\tau}}\right) x\left[\sqrt{t^{\prime}} u_{1}\right] \Phi\left[x\left[\sqrt{t^{\prime}} u_{1}+\sqrt{\tau} u_{2}\right]\right]
\end{aligned}
$$

One finds:

$$
\begin{aligned}
& X_{C}=\frac{1}{T} \frac{D}{A+D} \\
& X_{B}=-\frac{1}{2 T} \frac{D}{D+A-A_{0}}
\end{aligned}
$$

where $A_{0}=A\left(t^{\prime}, 0\right)$.

Thus one gets for instance:

$$
X_{B} \sim-\frac{x}{2 T} \frac{<\epsilon\left(u_{1}\right) \epsilon\left(u_{1}+x u_{2}\right) \ln \left(1+\sqrt{t^{\prime}}\left|u_{1}+x u_{2}\right|\right)-<\ln \left(1+\sqrt{t^{\prime}}\left|u_{1}\right|\right)}{<\frac{x u_{1}-u_{2}}{u_{1}+x u_{2}} \epsilon\left(u_{1}\right) \epsilon\left(u_{1}+x u_{2}\right) \ln \left(1+\sqrt{t^{\prime}}\left|u_{1}+x u_{2}\right|\right)}
$$

with $x=\sqrt{\tau} t^{\prime}$. The divergences make the calculation depend strongly on the boundary conditions. As explained in the text we have not pursued it further.

\section{APPENDIX F: DIRECTED MODEL CALCULATIONS}

Defining:

$$
\Phi(s)=\overline{\frac{1}{s+W}}
$$

We will use that:

$$
\begin{gathered}
\frac{\bar{W}}{s+W}=1-s \Phi(s) \\
\frac{1}{\frac{\left(s_{1}+W\right)\left(s_{2}+W\right)}{W}}=\frac{\Phi\left(s_{1}\right)-\Phi\left(s_{2}\right)}{s_{2}-s_{1}} \\
\frac{s_{2} \Phi\left(s_{2}\right)-s_{1} \Phi\left(s_{1}\right)}{s_{2}-s_{1}}
\end{gathered}
$$


Let us compute the averaged probability that the particle advances by $m$ between $t^{\prime}$ and $t=t^{\prime}+\tau$ :

$$
Q\left(m, \tau, t^{\prime}\right)=\left\langle\overline{\delta\left(x(t)-x\left(t^{\prime}\right)-m\right)}\right\rangle=\sum_{n \geq 0} \overline{P(n+m, n, \tau) P\left(n, 0, t^{\prime}\right)}
$$

The double LT, $Q\left(m, s_{1}, s_{2}\right)=\int_{0}^{\infty} \int_{0}^{\infty} d \tau d t^{\prime} e^{-s_{1} \tau-s_{2} t^{\prime}} P\left(m, \tau, t^{\prime}\right)$ can be calculated:

$$
\begin{aligned}
Q\left(m, s_{1}, s_{2}\right)= & \sum_{n \geq 0} \overline{P\left(n+m, n, s_{1}\right) P\left(n, 0, s_{2}\right)}=\left(\delta_{m 0}\left(\frac{1}{\left(s_{1}+W\right)\left(s_{2}+W\right)}\right)\right. \\
& +\left(1-\delta_{m 0}\right)\left(\overline{\frac{1}{s_{1}+W}}\right)\left(\frac{W}{\left.\left(\frac{W}{\left.s_{1}+W\right)\left(s_{2}+W\right)}\right)\left(\frac{W}{s_{1}+W}\right)^{m-1}\right) \sum_{n=0}^{\infty} \overline{\left(\frac{W}{s_{2}+W}\right)^{n}}}\right.
\end{aligned}
$$

It yields the result given in the text.

Let us now estimate the probability $P_{t_{w}}(W)$ that at time $t_{w}$ the walker is on a site with a waiting time $W=1 / \tau$. Its Laplace transform with respect to $t_{w}$ (Laplace variable $s_{2}$ ) is simply given by:

$$
\overline{\sum_{n} \delta\left(W-W_{n}\right) \frac{1}{s_{2}+W_{n}} \prod_{k=n_{0}}^{n-1} \frac{W_{k}}{s_{2}+W_{k}}}
$$

This easily leads to:

$$
\frac{1}{s_{2}} P(W) \frac{1}{s_{2}+W} \frac{1}{\Phi\left(s_{2}\right)}
$$

By Laplace inversion this yields for the distribution of the waiting time $\tilde{\tau}=1 / W$ :

$$
P_{t_{w}}(\tilde{\tau}) d \tilde{\tau}=\frac{\sin (\pi \mu)}{\pi} \frac{d \tilde{\tau}}{t_{w}}\left(\frac{t_{w}}{\tilde{\tau}}\right)^{1+\mu} \int_{0}^{1} e^{-\frac{t_{w}}{\tau}(1-u)} \frac{u^{\mu-1}}{\Gamma[\mu]}
$$


${ }^{1}$ Unité associée du CNRS (URA 280), Université Paris 6.

${ }^{2}$ Laboratoire propre du CNRS associée à l'ENS et à l'Université Paris-Sud.

${ }^{3}$ A. J. Bray Adv. Phys. 43357 (1994).

${ }^{4}$ E. Vincent, J. Hammann e M. Ocio; in 'Recent progress in Random Magnets', ed. D. H. Ryan, World Scientific, Singapore (1992).

${ }^{5}$ M. Alba, M. Ocio, J. Hammann, Europhys. Lett. 242 (1986).

${ }^{6}$ H. Rieger; J. Phys. A26, L615 (1993).

${ }^{7}$ H. Rieger, cond-mat 9402109 and cond-mat 9411017 to appear in Annual Reviews of Computational Physics.

${ }^{8}$ D.S. Fisher, D.A. Huse Phys. Rev. Lett. 561601 (1986), Phys. Rev. B 38373.

${ }^{9}$ P. Sibani Phys. Rev. B 358572 (1987) K. H. Hoffmann and P. Sibani Z. Phys. B 80429 (1990)

${ }^{10}$ A. Barrat, R. Burioni, M. Mezard, Preprint cond-mat 9509142 submitted to J. Phys. A. (1995).

11 J. Kurchan and L. Laloux J. Phys. A Math. Gen. 291929 (1996).

12 G. Blatter et al., Rev. Mod. Phys. 661125 (1994).

13 T. Giamarchi and P. Le Doussal Phys. Rev. B52 1242 (1995).

${ }^{14}$ P. Le Doussal and T. Giamarchi; Phys. Rev. Lett. 74, 606 (1995). J. Kierfeld; J. Phys. I 5, 379 (1995).

15 T. Giamarchi and P. Le Doussal Preprint cond-mat/9705096

${ }^{16}$ L. C. E. Struik; 'Physical aging in amorphous polymers and other materials', Elsevier, Houston (1978).

${ }^{17}$ V. Dotsenko, M. Feigel'man and I. Ioffe; Spin glasses and related problems Soviet Scientific Reviews 15 (New York: Harwood Academic).

${ }^{18}$ W. Götze; in Liquids, freezing and the glass transition, J. P. Hansen, D. Levesque and J. Zinn-Justin eds. (Les Houches Session LI, Elsevier Sc. Publishers BV, 1991).

${ }^{19}$ L. F. Cugliandolo and J. Kurchan; Phys. Rev. Lett. 71, 173 (1993).

L. F. Cugliandolo and J. Kurchan; J. Phys. A27, 5749 (1994).

${ }^{20}$ E Vincent, J Hammann, M Ocio, J-P Bouchaud and L F Cugliandolo, Slow dynamics and aging, Proceedings form the Sitges conf., ed E rubi, (springer-verlag) cond-mat/9607224

${ }^{21}$ L. Laloux, Thèse de doctorat de l'Universite PM Curie (Paris 6) 20 December 1996.

${ }^{22}$ B. Derrida J. Stat. Phys. 31433 (1983)

${ }^{23}$ M. Feigelman and V. M. Vinokur, J. Phys. France 491731 (1988)

${ }^{24}$ F. Solomon, Ann. Probab. 31 (1975), H. Kesten, M. Koslov, F. Spitzer, Compos. Math. 30145 (1975)

${ }^{25}$ B. Derrida and Y. Pomeau, Phys. Rev. Lett. 48627 (1982).

${ }^{26}$ Y. G. Sinai Theor. Probab. Its Appl. 27247 (1982)

27 A. O. Golosov Soviet. Math. Dokl. 2819 (1983).

28 J. P. Bouchaud, A. Comtet, A. Georges and P. Le Doussal Europhys. Lett. 3653 (1987).

29 J. P. Bouchaud, A. Comtet, A. Georges and P. Le Doussal, Ann Phys. 201285 (1990)

${ }^{30}$ J.P. Bouchaud, A. Comtet, A. Georges, P. Le Doussal, J. Physique (Paris) 481445 (1987) and 49369 (1988)

${ }^{31}$ P. Le Doussal, J. Machta Phys. Rev. B 409427 (1989).

32 P. Le Doussal, Phys. Rev. Lett. 623097 (1989)

${ }^{33} \mathrm{P}$. Le Doussal, Thèse de Doctorat de l'Université Paris 6 (1987).

${ }^{34}$ Enzo Marinari and Giorgio Parisi, cond-mat/9308003

${ }^{35}$ L. F. Cugliandolo, J. Kurchan, P. Le Doussal, L. Peliti, preprint cond-mat/9606060 Phys. Rev. Lett. 78 350 (1997), P. Le Doussal, L. F. Cugliandolo, L. Peliti preprint cond-mat/9612079 submitted to Europhys. Lett. (1997).

${ }^{36}$ L. F. Cugliandolo and P. Le Doussal; cond-mat/9505112, Phys. Rev. E 531525 (1996).

${ }^{37}$ L. F. Cugliandolo, J. Kurchan and P. Le Doussal cond-mat/9509009 Phys. Rev. Lett. 762390 (1996).

${ }^{38}$ H. Kinzelbach and H. Horner; J. Phys. I (France) 3, 1329 (1993), ibid 3, 1901 (1993).

39 J.-P. Bouchaud; J. Phys. I (France) 2, 1705 (1992).

${ }^{40}$ L. Cugliandolo, D. Dean, J. Kurchan, preprint cond-mat/9705002

41 J.-P. Bouchaud and D.S. Dean; J. Phys. I (France) 5, 265 (1995).

${ }^{42}$ P. Le Doussal, Phys. Rev. Lett. 623097 (1989)

${ }^{43}$ Durett, Comm. Math. Phys. 10487 (1986).

${ }^{44}$ H. Kesten Physica 138 A 299 (1986).

${ }^{45}$ M. Nauenberg, J. Stat. Phys. 41803 (1985).

${ }^{46}$ P. Le Doussal, V.M. Vinokur, cond-mat/9501131, Physica C Superconductivity 25463 (1995).

${ }^{47}$ K. Broderix, R. Kree preprint cond-mat/9507099 